Collection SFN 7 (2007) 123-145

(C) EDP Sciences, Les Ulis

DOI: $10.1051 / \mathrm{sfn}: 20070020$

\title{
Utilisations de l'analyse de polarisation pour la détermination de structures magnétiques
}

\author{
P.J. Brown ${ }^{1}$
}

\author{
${ }^{1}$ Institut Laue Langevin, BP. 156X, 38042 Grenoble Cedex, France \\ et
}

Physics Department, Loughborough University, Loughborough, UK

Résumé. Ce cours traite de l'application de la polarimétrie, et en particulier de la polarimétrie sphérique de neutrons (PSN) pour la détermination de structures magnétiques. La première partie nous permettra d'introduire quelques unes des équations fondamentales et de montrer comment ces équations sont reliées aux quantités mesurées lors de l'utilisation de l'analyse de polarisation. Ensuite, les problèmes qui pourraient être rencontrés lors de l'étude de cristaux réels seront introduits. Les dernières sections présentent des exemples de problèmes auxquels la PSN a été appliquée avec succès.

\section{NOTATIONS}

P Vecteur de polarisation incidente

$\mathrm{P}_{i} \quad i^{\text {ième }}$ composante de la polarisation incidente

$\mathbf{P}^{\prime} \quad$ Vecteur de polarisation diffractée

$\mathrm{P}_{i}^{\prime} \quad i^{\text {ième }}$ composante de la polarisation diffractée

$\mathbf{P}^{\prime \prime} \quad$ Polarisation crée par diffraction

$\mathrm{P}_{i}^{\prime \prime} \quad i^{\text {ième }}$ composante de la polarisation crée par diffraction

$\mathrm{P} \quad$ Matrice de polarisation

$\mathcal{P} \quad$ Tenseur de polarisation

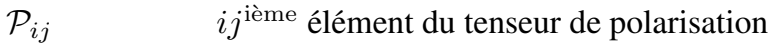

$F_{N}(\mathbf{Q}) \quad$ Facteur de structure nucléaire pour une réflexion en $\mathbf{Q}$

$F_{N} \quad$ Abréviation pour $F_{N}(\mathbf{Q})$

$F_{N}^{*} \quad$ Complexe conjugué de $F_{N}$

$F_{N}^{*}(\mathbf{Q}) \quad$ Complexe conjugué de $F_{N}(\mathbf{Q})$

$\mathbf{F}_{\mathbf{M}}(\mathbf{Q}) \quad$ Facteur de structure magnétique pour une réflexion en $\mathbf{Q}$

$\mathbf{M}_{\perp}(\mathbf{Q}) \quad$ Vecteur d'interaction magnétique pour une réflexion en $\mathbf{Q}$

$\mathbf{M}_{\perp}^{*}(\mathbf{Q}) \quad$ Complexe conjugué de $\mathbf{M}_{\perp}(\mathbf{Q})$

$\mathbf{M}_{\perp}$

Abréviation pour $\mathbf{M}_{\perp}(\mathbf{Q})$

$\mathbf{M}_{\perp}^{*} \quad$ Complexe conjugué de $\mathbf{M}_{\perp}$

$\mathrm{M}_{\perp i} \quad i^{\text {ième }}$ composante du vecteur d'interaction magnétique

$\mathrm{M}_{\perp} \quad$ Module du vecteur d'interaction magnétique

$\tau \quad$ Vecteur de propagation magnétique

M(r) Distribution d'aimantation 


$\begin{array}{ll}\mathbf{g} & \text { Vecteur du réseau réciproque } \\ l & \text { Vecteur du réseau cristallin } \\ \mathbf{r} & \text { Vecteur radial dans l'espace réel } \\ \mathbf{Q} & \text { Vecteur de diffusion }=k_{i}-k_{f} \\ \kappa & \text { Vecteur de diffusion cristallographique }=-\mathbf{Q}\end{array}$

\section{PROPRIÉTÉS VECTORIELLES DE LA DIFFUSION DE NEUTRONS}

\subsection{Le vecteur d'interaction magnétique}

Considérons uniquement l'interaction dipole-dipole entre le spin du neutron et un champ vectoriel $\mathbf{A}(\mathbf{r})$. Si le vecteur de polarisation incidente est $\mathbf{P}, \mathbf{P}^{\prime}$ est le vecteur de polarisation diffracté avec le vecteur de diffusion $\mathbf{Q}$ et $I$ est proportionelle à l'intensité diffusée, alors

$$
\begin{aligned}
\mathbf{P}^{\prime} I & =\mathbf{A}(\mathbf{Q})\left(\mathbf{P} \cdot \mathbf{A}^{*}(\mathbf{Q})\right)+\mathbf{A}^{*}(\mathbf{Q})(\mathbf{P} \cdot \mathbf{A}(\mathbf{Q})) \\
& -\mathbf{P}\left(\mathbf{A}(\mathbf{Q}) \cdot \mathbf{A}^{*}(\mathbf{Q})\right)+i\left(\mathbf{A}(\mathbf{Q}) \times \mathbf{A}^{*}(\mathbf{Q})\right)
\end{aligned}
$$

et

$$
I=\mathbf{A}(\mathbf{Q}) \cdot \mathbf{A}^{*}(\mathbf{Q})-i \mathbf{P} \cdot \mathbf{A}(\mathbf{Q}) \times \mathbf{A}^{*}(\mathbf{Q})
$$

où $\mathbf{A}(\mathbf{Q})$ est la $Q^{\text {ième }}$ composante de Fourier du champ vectoriel $\mathbf{A}(\mathbf{r})$

$$
\mathbf{A}(\mathbf{Q})=\int \mathbf{A}(\mathbf{r}) \exp (-i \mathbf{Q} \cdot \mathbf{r}) d r^{3}
$$

Dans le cas de la diffraction magnétique par un cristal, le champ vectoriel est l'induction magnétique $\mathbf{B}(\mathbf{r})=\mu_{\mathbf{0}} \mathbf{H}+\mathbf{M}(\mathbf{r})$ où $\mathbf{H}$ est le champ magnétique appliqué et $\mathbf{M}(\mathbf{r})$ est la distribution d'aimantation dans le cristal. La $Q^{\text {ième }}$ composante de Fourier est

$$
\mathbf{M}_{\perp}(\mathbf{Q})=\hat{\mathbf{Q}} \times \mathbf{F}_{\mathbf{M}}(\mathbf{Q}) \times \hat{\mathbf{Q}}
$$

avec $\mathbf{F}_{\mathbf{M}}(\mathbf{Q})=\int \mathbf{M}(\mathbf{r}) \exp (-i \mathbf{Q} \cdot \mathbf{r}) d r^{3}$.

$\hat{\mathbf{Q}}$ est un vecteur unitaire parallèle au vecteur de diffusion $\mathbf{Q}$.

$\mathbf{F}_{\mathbf{M}}(\mathbf{Q})$ est le facteur de structure magnétique, $\mathbf{M}_{\perp}(\mathbf{Q})$ est le vecteur d'interaction magnétique, les deux étant en général des vecteurs complexes.

L'équation 2.4 traduit que seules les composantes de l'aimantation perpendiculaires au vecteur de diffusion donne lieu à de la diffraction magnétique.

\subsection{Rotation de la polarisation par diffraction magnétique}

En termes de vecteur d'interaction magnétique, l'intensité diffractée est proportionelle à

$$
I=\mathbf{M}_{\perp}(\mathbf{Q}) \cdot \mathbf{M}_{\perp}^{*}(\mathbf{Q})-i \mathbf{P} \cdot \mathbf{M}_{\perp}(\mathbf{Q}) \times \mathbf{M}_{\perp}^{*}(\mathbf{Q})
$$

et la polarisation diffractée est donnée par

$$
\begin{aligned}
\mathbf{P}^{\prime} I= & 2 \Re\left(\mathbf{M}_{\perp}(\mathbf{Q})\left(\mathbf{P} \cdot \mathbf{M}_{\perp}^{*}(\mathbf{Q})\right)\right)+\mathbf{P}\left(\mathbf{M}_{\perp}(\mathbf{Q}) \cdot \mathbf{M}_{\perp}^{*}(\mathbf{Q})\right) \\
& +i\left(\mathbf{M}_{\perp}(\mathbf{Q}) \times \mathbf{M}_{\perp}^{*}(\mathbf{Q})\right)
\end{aligned}
$$


Lorsque $\mathbf{M}_{\perp}(\mathbf{Q})$ et $\mathbf{M}_{\perp}^{*}(\mathbf{Q})$ sont parallèles, la polarisation précesse de $180^{\circ}$ autour de $\mathbf{M}_{\perp}(\mathbf{Q})$ comme illustré sur la Figure 1(a). Lorsque $\mathbf{M}_{\perp}(\mathbf{Q})$ et $\mathbf{M}_{\perp}^{*}(\mathbf{Q})$ sont perpendiculaires, la polarisation diffractée est perpendiculaire à chacun d'eux comme présenté sur la Figure 1(b).

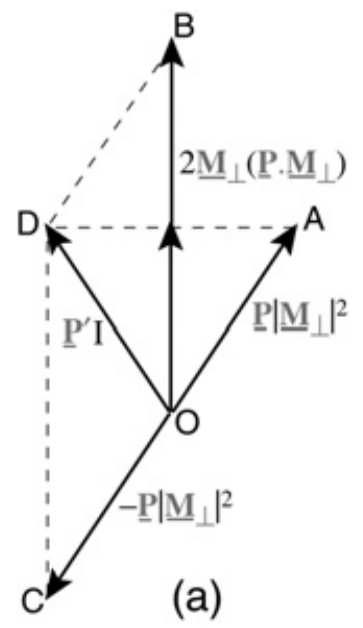

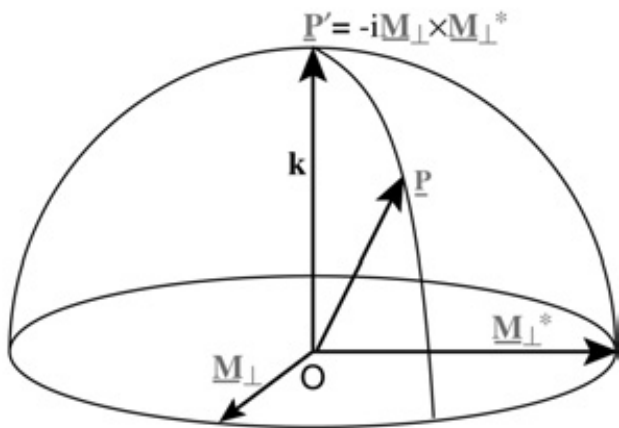

(b)

Figure 1. Rotation de la polarisation par diffraction purement magnétique ; (a) quand $\mathbf{M}_{\perp}$ et $\mathbf{M}_{\perp}^{*}$ sont parallèles, (b) quand $\mathbf{M}_{\perp}$ et $\mathbf{M}_{\perp}^{*}$ sont perpendiculaires.

\subsection{Interférence nucleaire magnétique}

Considérons en premier lieu uniquement la diffraction nucléaire et supposons que tous les spins nucléaires sont orientés aléatoirement. Alors, l'interaction entre le neutron et le noyau peut être approximée par le pseudo potentiel de Fermi qui est un champ scalaire. Il est nul sauf très proche du noyau. Pour cette raison, la diffraction nucléaire pure (spins nucléaires aléatoires) est indépendante de la polarisation du neutron.

$$
\mathbf{P}^{\prime} I=\mathbf{P}\left(F_{N}(\mathbf{Q}) F_{N}^{*}(\mathbf{Q})\right) \quad \text { et } \quad I=F_{N}(\mathbf{Q}) F_{N}^{*}(\mathbf{Q})
$$

$F_{N}(\mathbf{Q})$ est le facteur de structure nucléaire, une quantité scalaire complexe. La diffusion cohérente nucléaire n'altère la polarisation ni en amplitude, ni en direction. En revanche, lorsque les diffractions magnétique et nucléaire se produisent sur les même réflexions (même $\mathbf{Q}$ ), leur interférence affecte à la fois l'intensité diffractée et la polarisation. Le terme additionnel de l'intensité est

$$
\mathbf{P} \cdot \mathbf{2} \Re\left(\mathbf{M}_{\perp}(\mathbf{Q}) F_{N}^{*}(\mathbf{Q})\right)
$$

Il est proportionnel à la projection, dans l'espace vectoriel complexe, de $\mathbf{M}_{\perp}(\mathbf{Q})$ sur $\mathbf{P} F_{N}(\mathbf{Q})$.

Il existe deux termes supplémentaires dans la polarisation, le premier

$$
2 \Re\left(\mathbf{M}_{\perp}(\mathbf{Q}) F_{N}^{*}(\mathbf{Q})\right)
$$

est présent uniquement lorsque l'intensité dépend de la polarisation comme ci-dessus. Le econd

$$
\mathbf{P} \times \mathbf{2} \Im\left(\mathbf{M}_{\perp}(\mathbf{Q}) F_{N}^{*}(\mathbf{Q})\right)
$$

n'est présent que s'il existe une composante de $\mathbf{M}_{\perp}(\mathbf{Q})$, dans l'espace vectoriel complexe, perpendiculaire à $\mathbf{P} F_{N}(\mathbf{Q})$. Ceci se produit lorsque $\mathbf{P}$ n'est pas parallèle à $\mathbf{M}_{\perp}(\mathbf{Q})$ et que la différence de phase entre $\mathbf{M}_{\perp}(\mathbf{Q})$ et $F_{N}(\mathbf{Q})$ n'est ni de 0 , ni de $180^{\circ}$. 
L'interférence nucléaire magnétique peut alors modifier à la fois l'amplitude et la direction de la polarisation. Ceci est illustré schématiquement sur la Figure 2. En (a) $\mathbf{M}_{\perp}(\mathbf{Q})$ et $F_{N}(\mathbf{Q})$ sont tous les deux réels et la polarisation tourne dans le plan contenant $\mathbf{P}$ and $\mathbf{M}_{\perp}(\mathbf{Q})$. En (b) $\mathbf{M}_{\perp}(\mathbf{Q})$ et $F_{N}(\mathbf{Q})$ sont en quadrature et la polarisation diffractée tourne vers $\mathbf{P} \times \mathbf{M}_{\perp}(\mathbf{Q})$.
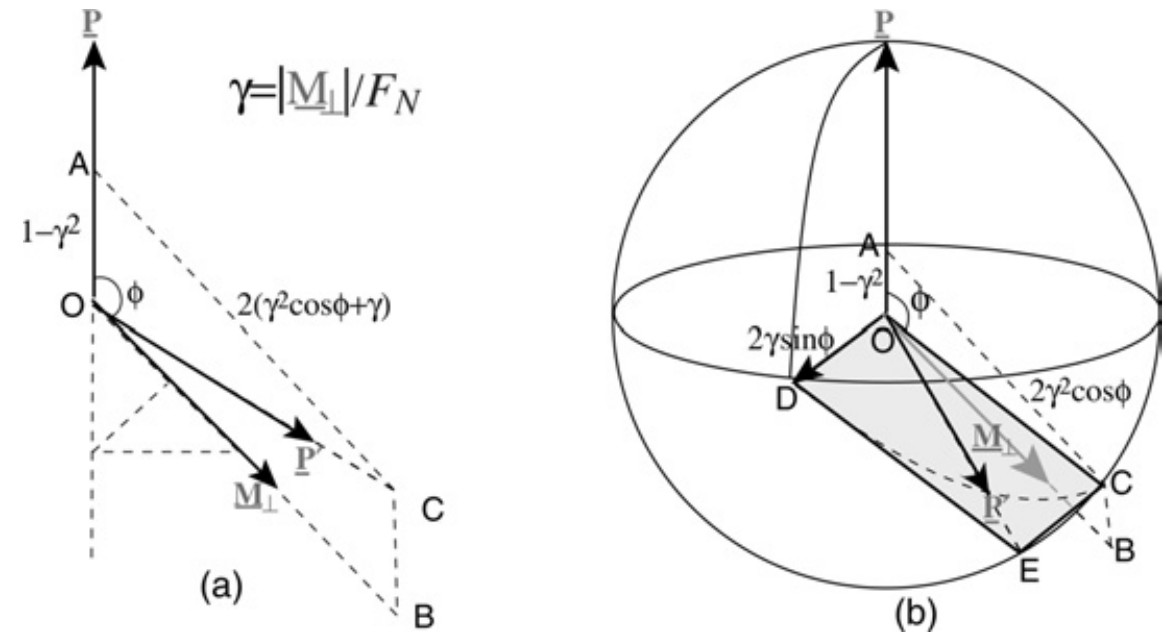

(b)

Figure 2. Rotation de la polarisation lorsque les diffractions magnétique et nucléaire se produisent ensemble ; (a) si $\mathbf{M}_{\perp}(\mathbf{Q})$ et $F_{N}(\mathbf{Q})$ sont tous les deux réels, (b) si $\mathbf{M}_{\perp}(\mathbf{Q})$ et $F_{N}(\mathbf{Q})$ sont en quadrature.

\section{4 Équations de Blume Maleev}

La combinaison des différentes contributions à l'intensité et à la polarisation à partir des équations 2.5-2.10 permet d'obtenir les équations de Blume-Maleev [1,2]. Elles donnent l'intensité diffractée I et la polarisation $\mathbf{P}^{\prime}$ en termes de polarisation incidente $\mathbf{P}$.

$$
\begin{aligned}
\mathbf{P}^{\prime} I= & \mathbf{P}\left(\left|F_{N}\right|^{2}-\mathbf{M}_{\perp} \cdot \mathbf{M}_{\perp}^{*}\right) \\
& +2 \Re\left(\mathbf{M}_{\perp}\left(\mathbf{P} \cdot \mathbf{M}_{\perp}^{*}\right)+\mathbf{M}_{\perp} F_{N}^{*}\right) \\
& +\mathbf{P} \times 2 \Im\left(\mathbf{M}_{\perp} F_{N}^{*}\right) \\
& -\Im\left(\mathbf{M}_{\perp} \times \mathbf{M}_{\perp}^{*}\right) \\
I \quad & \left|F_{N}\right|^{2}+\mathbf{M}_{\perp} \cdot \mathbf{M}_{\perp}^{*} \\
& +2 \Re\left(\mathbf{P} \cdot \mathbf{M}_{\perp} F_{N}^{*}\right)+\mathbf{P} \cdot \Im\left(\mathbf{M}_{\perp} \times \mathbf{M}_{\perp}^{*}\right)
\end{aligned}
$$

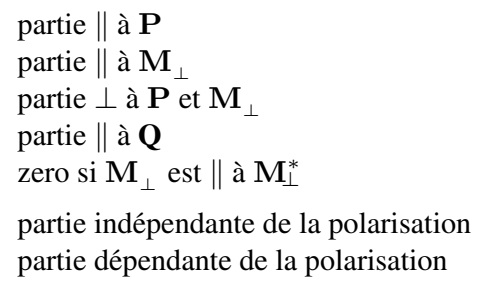

partie indépendante de la polarisation partie dépendante de la polarisation

La polarisation tourne lors du processus de diffraction à chaque fois que le vecteur d'interaction magnétique n'est pas parallèle à la polarisation incidente.

\subsection{Représentation tensorielle de la polarisation diffractée}

La relation entre les polarisations incidente et diffractée peut être commodément décrite par une équation tensorielle.

$$
\mathbf{P}^{\prime}=\mathcal{P} \mathbf{P}+\mathbf{P}^{\prime \prime} \quad \text { ou en composantes } \quad \mathbf{P}_{i}^{\prime}=\mathcal{P}_{i j} \mathbf{P}_{j}+\mathbf{P}_{i}^{\prime \prime}
$$

$\mathbf{P}^{\prime \prime}$ est la polarisation créee lors du processus de diffraction. 
L'unique direction définie par le processus de diffraction est celle du vecteur de diffraction $\mathbf{Q}$. L'expérience définie habituellement une seconde direction spéciale : la normale au plan de diffraction. Il est donc pratique de travailler avec des axes définis par rapport à ces directions :

$x$ parallèle au vecteur de diffraction cristallographique $\kappa=-\mathbf{Q}$

$z$ normal au plan de diffraction

y complétant le référentiel orthogonal trigonométrique

Avec cette définition, il n'existe pas de composante de $\mathbf{M}_{\perp}(\mathbf{Q})$ parallèle à $x$. Ces axes sont ceux de la polarisation. Les éléments de $\mathcal{P}$ et $\mathbf{P}^{\prime \prime}$ peuvent être écris dans le référentiel de ces axes comme :

$$
\begin{aligned}
& \mathcal{P}=\left(\begin{array}{lll}
\left(F_{N}^{2}-M_{\perp}{ }^{2}\right) / I_{x} & J_{n z} / I_{x} & J_{n y} / I_{x} \\
-J_{n z} / I_{y} & \left(F_{N}^{2}-M_{\perp}{ }^{2}+R_{y y}\right) / I_{y} & R_{y z} / I_{y} \\
-J_{n y} / I_{z} & \left(F_{N}^{2}-M_{\perp}{ }^{2}+R_{z z}\right) / I_{z}
\end{array}\right) \\
& \mathbf{P}^{\prime \prime}=\left(\begin{array}{c}
-J_{y z} / I \\
R_{n y} / I \\
R_{n z} / I
\end{array}\right) \\
& R_{z y} / I_{z} \\
& I_{x}=F_{N}^{2}+M_{\perp}{ }^{2}+P_{x} J_{y z} \\
& I_{z}=F_{N}^{2}+M_{\perp}{ }^{2}+P_{z} R_{n z} \\
& I=F_{N}^{2}+M_{\perp}{ }^{2}+P_{x} J_{y z}+P_{y} R_{n y}+P_{z} R_{n z} \\
& F_{N}^{2}=F_{N}(\mathbf{Q}) F_{N}^{*}(\mathbf{Q}) M_{\perp}{ }^{2}=\mathbf{M}_{\perp}(\mathbf{Q}) \cdot \mathbf{M}_{\perp}^{*}(\mathbf{Q}) \\
& R_{i j}=2 \Re\left(M_{\perp i}(\mathbf{Q}) M_{\perp j}^{*}(\mathbf{Q})\right) R_{n i}=2 \Re\left(F_{N}(\mathbf{Q}) M_{\perp i}^{*}(\mathbf{Q})\right) \\
& J_{i j}=2 \Im\left(M_{\perp i}(\mathbf{Q}) M_{\perp j}^{*}(\mathbf{Q})\right) J_{n i}=2 \Im\left(F_{N}(\mathbf{Q}) M_{\perp i}^{*}(\mathbf{Q})\right)
\end{aligned}
$$

Les composantes non-diagonales de $\mathcal{P}$ décrivent les rotations de la polarisation.

\section{DIFFRACTION PAR DES CRISTAUX RÉELS : DOMAINES MAGNÉTIQUES}

Le carré du module de la polarisation diffractée $\left|\mathbf{P}^{\prime}\right|^{2}$, calculé à partir des équations de Blume-Maleev, est toujours supérieur ou égal à $|\mathbf{P}|^{2}$.

$$
1 \geq\left|\mathbf{P}^{\prime}\right|^{2} \geq|\mathbf{P}|^{2}
$$

La polarisation du faisceau est ainsi soit augmentée soit inchangée lors de la diffraction par un état pur.

Néanmoins, un faisceau polarisé diffracté par un cristal réel est souvent au moins partiellement dépolarisé. En effet, les cristaux réels contiennent un mélange d'états correspondants généralement à différents domaines magnétiques. La PSN permet de distinguer une dépolarisation réelle, due à la présence de plusieurs domaines, d'une rotation de la polarisation par rapport à la direction de la polarisation incidente car elle permet de déterminer les éléments non-diagonaux de la matrice de polarisation.

Les domaines magnétiques existent lorsque la symétrie de la structure magnétique est plus basse que celle de la phase paramagnétique. Si l'ordre du groupe paramagnétique est $p$ et celui du groupe magnétique $m$; le nombre de domaines différents est $p / m$.

\subsection{Domaines de configuration}

Les domaines de configuration existent lorsque le vecteur de propagation $\tau$ décrivant la structure magnétique n'est transformé ni en lui même, ni en lui même plus un vecteur du réseau réciproque, par tous les opérateurs de symétrie du groupe paramagnétique. Les opérateurs de symétrie de l'état paramagnétique appliqués à $\tau$ génèrent un ensemble de vecteurs qui forme l'étoile des $\tau$. Chaque vecteur de 
l'étoile est à l'origine d'un domaine de configuration et chaque domaine de configuration donne lieu à un ensemble distinct de reflexions magnétiques, comme illustré sur la Figure 3 pour $\tau=\frac{1}{2} \frac{1}{2} 0$ et le groupe d'espace $P 4 / \mathrm{mmm}$. La Figure 3(a) montre les 4 bras de l'étoile générée par la symétrie tétragonale, dont seulement 2 sont indépendants puisque $2 \tau$ est un vecteur du réseau réciproque. La figure 3(b) donne la position des réflexions magnétiques dans le réseau réciproque pour les deux domaines de configuration.

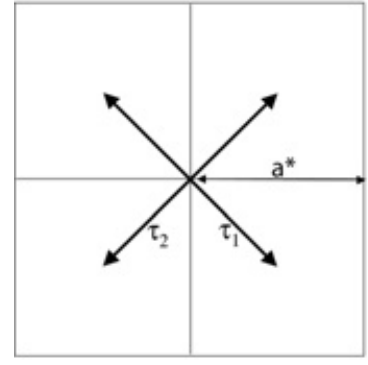

(a)

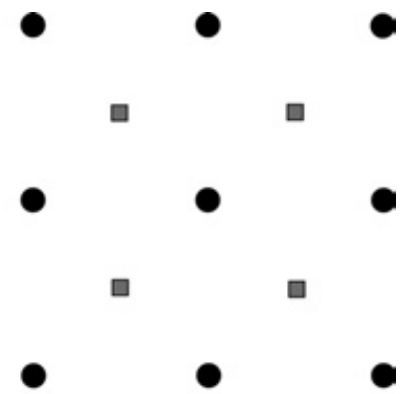

(b)

Figure 3. (a) L'étoile du vecteur de propagation $\tau$ générée par la symétrie tétragonale. (b) Positions dans le réseau réciproque des réflexions nucléaires (cercles) et magnétiques (carrés) correspondant à l'étoile complète.

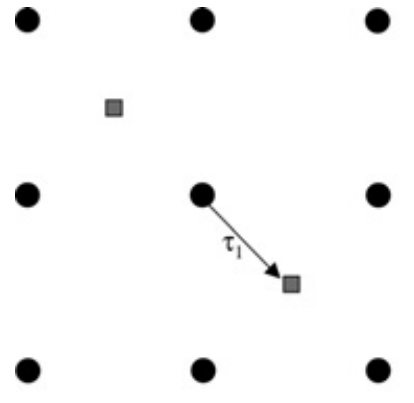

(a)

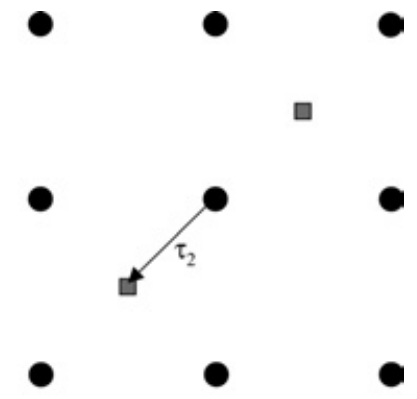

(b)

Figure 4. Positions, dans le réseau réciproque, des réflexions magnétiques appartenant aux deux domaines de configuration distincts.

La figure 4 montre la position, dans l'espace réciproque, des réflexions magnétiques pour chacun des deux domaines de configuration. Ces deux domaines correspondent à des vecteurs de propagation, $\tau_{1}$ et $\tau_{2}$, différents mais néanmoins équivalents. Chaque réflexion appartient à un domaine de configuration distinct et donc effectivement à un seul état. Pour cette raison, les domaines de configuration ne produisent pas de dépolarisation.

\subsection{Domaines à $\mathbf{1 8 0}^{\circ}$}

Les domaines à $180^{\circ}$ degrés sont des régions du cristal dans lesquelles toutes les directions des moments d'un des domaines sont renversées par rapport à celles de l'autre domaine. $\mathbf{M}_{\perp}$ est orienté selon des directions opposées dans les deux domaines ce qui implique une différence de phase de $\pi$ entre leurs amplitudes diffractées. Les deux domaines sont reliés par l'opérateur d'inversion du temps. Les domaines ferromagnétiques en sont un exemple simple. 


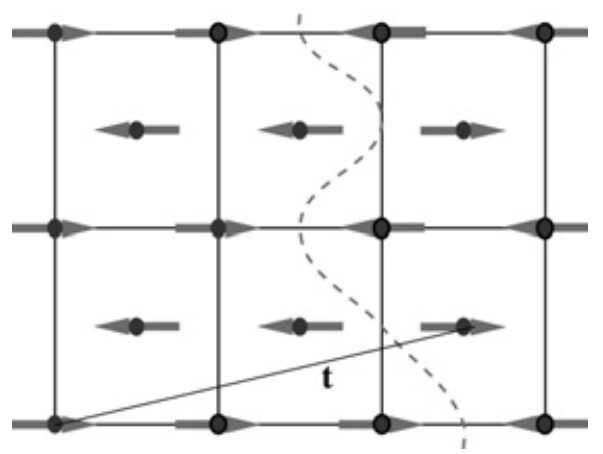

Figure 5. Domaines à $180^{\circ}$ dans un réseau face-centrée pour une structure magnétique caractérisée par $\tau=1,0,0$.

Dans une structure de vecteur de propagation non-nul $(\tau \neq 0)$, les deux domaines à $180^{\circ}$ ne peuvent pas être différenciés sauf par les défauts associés aux parois de domaines. Un domaine peut être transformé en l'autre par une translation $\mathbf{t}$ telle que $\mathbf{t} \cdot \boldsymbol{\tau}=(2 n+1) / 2$. L'intensité et la polarisation diffractées par les deux domaines sont identiques.

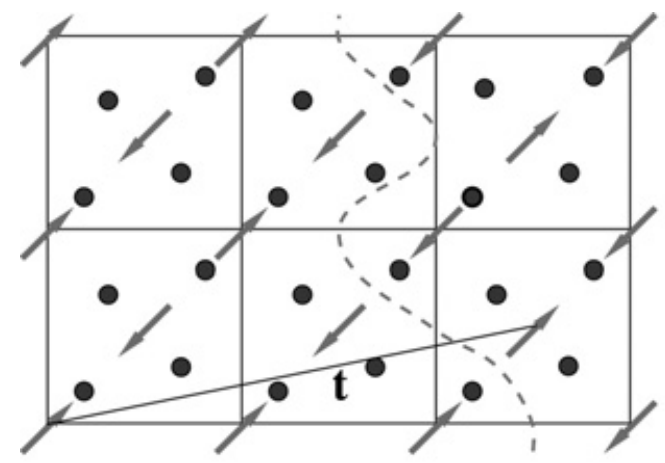

(a)

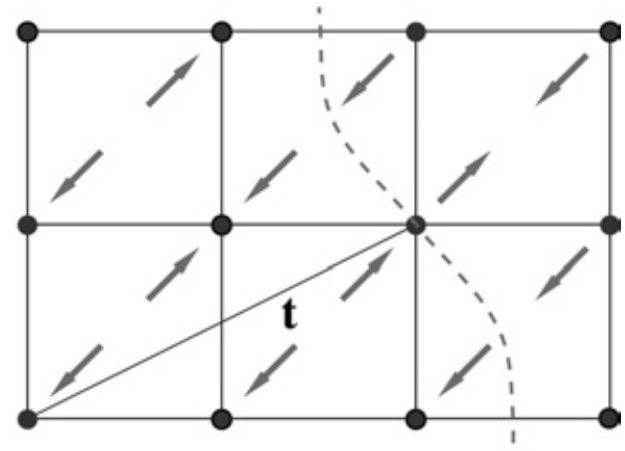

(b)

Figure 6. Domaines à $180^{\circ}$ dans des structures $\tau=0$ (a) lorsque l'élément de symétrie qui retourne les spins met en jeu une rotation propre, (b) lorsque la rotation est impropre ie qu'elle implique une inversion.

Lorsque $\tau=0$, une rotation et une translation sont requises afin de transformer un domaine à $180^{\circ}$ en l'autre. La Figure 6(a) montre un exemple de structure centro-symétrique dans laquelle des moments d'orientation opposée sont reliés entre eux par une rotation de $\pi / 2$ et une translation de $\left(\frac{1}{2} \frac{1}{2} 0\right)$. Les facteurs de structure magnétique et nucléaire sont tout deux réels de sorte que les termes $R_{n i}$, dans l'èquation 2.13, sont non-nuls. Ils pourraient donner lieu à une section efficace dépendante de la polarisation, et augmenter la polarisation parallèle à $\mathbf{M}_{\perp}$. Cependant, ces termes sont de signes opposés pour les deux domaines et si ces domaines sont également peuplés, les $R_{n i}$ se moyenneront à zéro. Il n'y a pas de dépolarisation due à ce type de domaines à $180^{\circ}$.

La Figure 6(b) présente quant à elle le cas d'une structure centro-symétrique dans laquelle les moments d'orientation opposée sont reliés par un centre de symétrie. Dans ce cas, les diffractions magnétique et nucléaire sont en quadrature de phase. Les termes $J_{n i}$ de l'équation 2.13 sont finis et induisent une rotation de la polarisation vers la direction perpendiculaire à $\mathbf{M}_{\perp}$ et $\mathbf{P}$. Ils sont, de plus, de signes opposés pour les deux domaines et, dans un cristal présentant un mélange de domaines, ils donneront lieu à de la dépolarisation à moins que la polarisation incidente ne soit parallèle à $\mathbf{M}_{\perp}$. 


\subsection{Domaines d'orientation (domaines-s)}

Les domaines d'orientation apparaissent lorsque le groupe d'espace magnétique n'est pas congruent avec le groupe décrivant la symétrie de configuration. Si $\mathcal{P}$ est un sous-groupe de la symétrie de configuration $\mathcal{G}$, congruent avec le groupe d'espace magnétique $\mathcal{M}$, les domaines d'orientation sont générés par le sous-groupe $\mathcal{S}$ de $\mathcal{G}$, constitué des opérateurs contenus dans $\mathcal{G}$ qui ne font pas partie du groupe magnétique. $\mathcal{S}$ est tel que

$$
\mathcal{G}=\mathcal{P} \times \mathcal{S} .
$$

Si $\mathcal{S}$ est d'ordre $s$, alors il y a $s$ domaines d'orientation possibles reliés les uns par rapport aux autres par les éléments de $\mathcal{S}$. Les vecteurs d'interaction magnétique pour les réflexions reliées par ces éléments sont différents.

$$
\mathbf{M}_{\perp}(\mathbf{Q}) \neq \mathbf{M}_{\perp}\left(\widetilde{\mathbf{R}}_{s} \mathbf{Q}\right) \quad \text { mais } \quad \mathbf{M}_{\perp} \mathbf{s}(\mathbf{Q})=\mathbf{M}_{\perp}\left(\widetilde{\mathbf{R}}_{\mathbf{s}} \mathbf{Q}\right)
$$

où $\widetilde{\mathbf{R}}_{s}$ est un opérateur de $\mathcal{S}$ et $\mathbf{M}_{\perp s}(\mathbf{Q})$ est le vecteur d'interaction pour le domaine généré par $\widetilde{\mathbf{R}}_{s}$. Pour des structures colinéaires, les facteurs de structure magnétique des réflexions reliées par les éléments de $\mathcal{S}$ sont égaux :

$$
\widetilde{\mathbf{R}}_{s} \mathbf{F}_{\mathbf{M}}(\mathbf{Q})=\mathbf{F}_{\mathbf{M}}\left(\widetilde{\mathbf{R}}_{s} \mathbf{Q}\right)
$$

mais cela n'est pas vrai dans le cas général.

Si la symétrie de configuration possède un axe de symétrie d'ordre supérieur à 2 , alors soit les moments sont parallèles à cet axe, soit la structure n'est pas colinéaire, soit l'axe de symétrie n'est pas dans le groupe d'espace magnétique. De même, dans une structure colinéaire, soit les moments sont parallèles à n'importe quel plan miroir et axe binaire, soit ils lui sont perpendiculaires, soit le plan miroir ou l'axe binaire n'est pas dans le groupe d'espace magnétique.

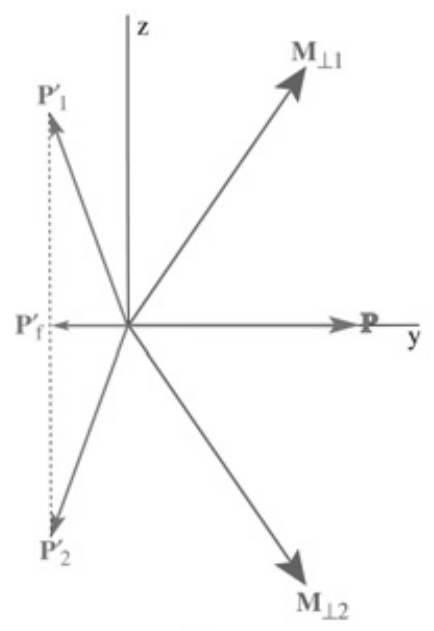

(a)

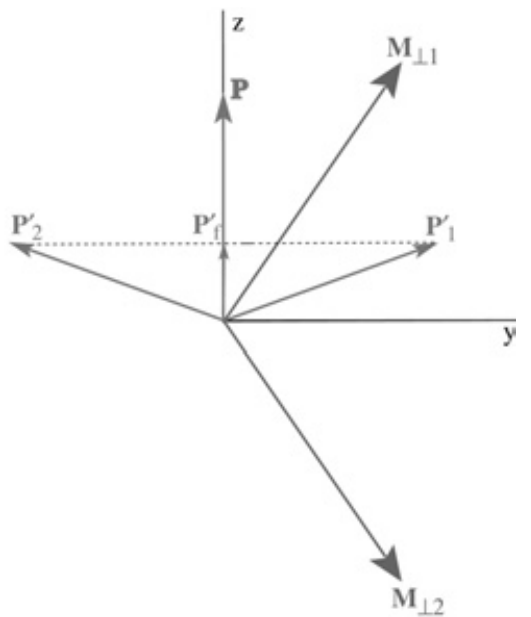

(b)

Figure 7. Rotation de la polarisation diffractée par des domaines d'orientation (a) domaines-s reliés par un axe binaire parallèle à $y$; (b) domaines-s reliés par un plan miroir perpendiculaire à $z$.

La Figure 7 illustre comment la présence de domaines-s peut dépolariser le faisceau. Sur la Figure 7(a), les domaines 1 et 2 , et leurs vecteurs d'interaction $\mathbf{M}_{\perp 1}$ et $\mathbf{M}_{\perp 2}$ sont reliés par un axe binaire parallèle à $y . \mathbf{P}^{\prime}{ }_{1}$ et $\mathbf{P}_{2}^{\prime}$ sont les directions de polarisations diffractées par les deux domaines quand $\mathbf{P}$ 
est parallèle à $y$; elles ont des composantes $z$ opposées. Dans un cristal où les domaines sont égaux, seules les composantes de la polarisation diffractée qui sont parallèles à $y$ (et $x$ ) sont conservées. Sur la Figure 7(b), les domaines 1 et 2 et leurs vecteurs d'interaction $\mathbf{M}_{\perp 1}$ et $\mathbf{M}_{\perp 2}$ sont reliés par un plan miroir parallèle à $z$. Dans ce cas, seules les composantes parallèles à $z$ (et $x$ ) de la polarisation diffractée seront conservées si les populations de domaines sont égales. De cette manière, les domaines d'orientation peuvent engendrer la dépolarisation des composantes $y$ et $z$ de la polarisation; la composante $x$ n'est quant à elle jamais dépolarisée. La connaissance des directions selon lesquelles a lieu la dépolarisation peut aider à identifier les éléments de symétrie manquants.

\subsection{Domaines de chiralité}

Les domaines de chiralité peuvent apparaître à chaque fois que le groupe d'espace paramagnétique est centro-symétrique mais que la structure magnétique ne l'est pas. Ils sont reliés entre eux par l'opérateur d'inversion. Cela se produit soit lorsque les moments magnétiques présents sur les sites reliés par centrosymétrie ne sont pas parallèles ou lorsque $2 \tau$ n'est pas un vecteur du réseau réciproque, le groupe de configuration étant alors non centro-symétrique. Dans ce dernier cas, les deux domaines de chiralité correspondent à + et $-\tau$. Tout deux donnent des réflexions en $\mathbf{g} \pm \boldsymbol{\tau}$ avec.

$$
\mathbf{M}_{\perp} \boldsymbol{\tau}(\mathbf{g}+\boldsymbol{\tau})=-\mathbf{M}_{\perp}^{*} \boldsymbol{\tau}(\mathbf{g}-\boldsymbol{\tau})=-\mathbf{M}_{\perp}{ }^{*} \boldsymbol{\tau}(g+\boldsymbol{\tau})
$$

De telles structures comprennent les hélices et les cycloïdes.

Dans les structures chirales, $\mathbf{M}_{\perp}(\mathbf{Q})$ n'est pas parallèle à $\mathbf{M}_{\perp}^{*}(\mathbf{Q})$ de sorte que les termes $J_{i j}$ de la matrice de polarisation sont non-nuls et sont de signes opposés pour les deux chiralités. Les domaines de chiralité ne donnent pas lieu à de la dépolarisation mais s'ils sont peuplés de façon inégale, la polarisation parallèle à $x$ peut augmenter et l'intensité va dépendre de la polarisation.

\section{DÉTERMINATION DE STRUCTURES MAGNÉTIQUES PAR PSN}

\subsection{Procédures expérimentales}

La PSN ne peut pas être utilisée seule pour déterminer une structure magnétique.

- Le vecteur de propagation magnétique $\tau$ doit être connu.

- Si $\tau \neq 0$, des mesures d'intensités sont nécessaires afin de déterminer les amplitudes absolues des moments.

- Dans la géométrie actuelle de la PSN, $\tau$ doit être positionné dans le plan de diffraction.

- Il est avantageux d'orienter le cristal de telle sorte qu'une composante de l'aimantation soit perpendiculaire au plan de diffraction.

- L'analyse d'un nombre relativement restreint de réflexions est alors généralement suffisante pour déterminer la structure.

La stratégie expérimentale habituelle consiste à mesurer la polarisation diffractée $\mathbf{P}^{\prime}$ en partant d'une polarisation incidente $\mathbf{P}$ parallèle à $x, y, z$ successivement. Cette démarche permet de déterminer la matrice de polarisation. La matrice de polarisation $\mathrm{P}$ est une quantité expérimentale reliée au tenseur de polarisation. L'élément de matrice $\mathrm{P}_{i j}$ donne la $j^{\text {ième }}$ composante de la polarisation diffractée lorsque la polarisation incidente est selon la $i^{\text {ième }}$ direction.

$$
\mathbf{P}_{i j}=\left\langle\frac{P_{i} \mathcal{P}_{i j}+P_{j}^{\prime \prime}}{P_{i}}\right\rangle_{\text {domaines }}
$$




\subsection{Structures commensurables de vecteurs de propagation non-nuls}

Pour des structures $\tau=0, F_{N}(\mathbf{Q})$ est nul aux positions des réflexions magnétiques. Ainsi, les termes $R_{n y}, R_{n z}, J_{n y}$ et $J_{n z}$ du tenseur de polarisation sont tous nuls. Si le groupe d'espace magnétique est centro-symétrique, $J_{y z}$ est également nul et donc $\mathbf{P}^{\prime \prime}=\mathbf{0}$. Les phases peuvent être choisies de sorte que $\mathbf{M}_{\perp}(\mathbf{Q})$ soit réel et s'il est incliné d'un angle $\alpha$ par rapport à $z$ (mesuré dans le sens des aiguilles d'une montre autour de $x$ ), le tenseur de polarisation peut s'écrire simplement :

$$
\mathcal{P}=\left(\begin{array}{ccc}
-1 & 0 & 0 \\
0 & -\cos 2 \alpha & -\sin 2 \alpha \\
0 & -\sin 2 \alpha & \cos 2 \alpha
\end{array}\right)
$$

Si $\mathbf{M}_{\perp}(\mathbf{Q})$ est parallèle à $y$ ou $z$, la matrice est diagonale.

$$
\mathcal{P}=\left(\begin{array}{ccc}
\left.\mathbf{M}_{\perp} \| y\right) & & (b) \\
0 & 1 & 0 \\
0 & 0 & -1
\end{array}\right) \quad \mathcal{P}=\left(\begin{array}{rrr}
-1 & 0 & 0 \\
0 & -1 & 0 \\
0 & 0 & 1
\end{array}\right)
$$

Lorsque des domaines-s avec des $\mathbf{M}_{\perp}(\mathbf{Q})$ de directions différentes sont présents, les éléments nondiagonaux $P_{y z}$ et $P_{z y}$ sont réduits et le faisceau diffracté est partiellement dépolarisé quand $\mathbf{P}$ n'est pas parallèle à $x$.

Le point fort de la PSN pour l'analyse des structures magnétiques commensurables est sa capacité à déterminer de façon univoque l'orientation de $\mathbf{M}_{\perp}(\mathbf{Q})$.

La structure commensurable de l'oxide de cuivre

La structure commensurable de l'oxide de cuivre est un exemple simple.

- En dessous de $213 \mathrm{~K}$, l'oxide de cuivre $\mathrm{CuO}$ (groupe d'espace $\mathrm{C} 2 / \mathrm{c}$ ) présente une structure antiferromagnétique commensurable définie par $\tau=\frac{1}{2}, 0,-\frac{1}{2}$.

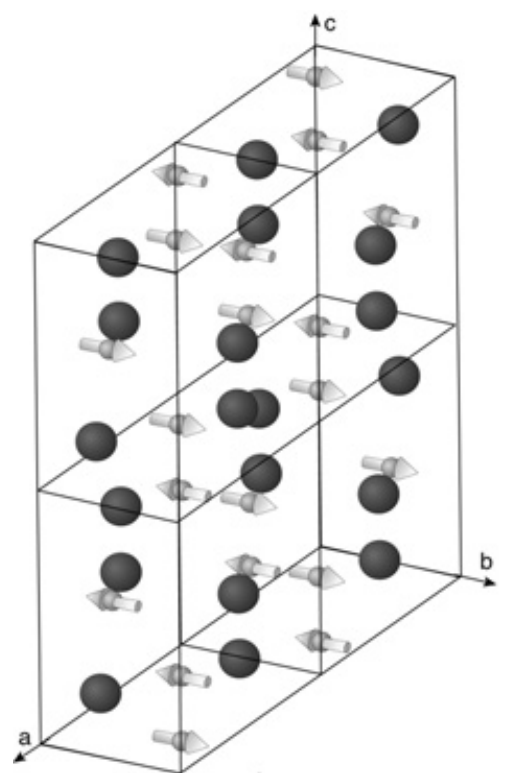

Figure 8. Structure magnétique de la phase commensurable de $\mathrm{CuO}$. 
- Les réflexions magnétiques $h 0 l \pm \tau$, avec $h+l$ impair, sont systématiquement absentes traduisant le fait que les spins, présents sur les atomes de cuivre couplés par le plan de glissement $n$ perpendiculaire à b, sont parallèles.

- Les intensités des réflexions suggèrent que la direction des spins est selon b [3].

Des mesures de PSN ont été entreprises afin de confirmer cette structure [5].

Le cristal était aligné avec la direction [010] perpendiculaire au plan de diffraction de sorte que les réflexions $h 0 l$ étaient accessibles. Les matrices de polarisation mesurées pour les réflexions $\frac{1}{2}, 0, \frac{1}{2} \quad \overline{\frac{1}{2}}$, $0, \frac{1}{2} \frac{3}{2}, 0, \frac{\overline{3}}{2}$ et $\frac{3}{2}, 0, \frac{1}{2}$ ont été trouvées identiques aux barres d'erreurs expérimentales près et présentant toutes la forme diagonale de l'équation 4.3(b). Ceci montre du manière concluante que la structure doit être colinéaire avec des spins parallèles à la polarisation $z=[010]$ comme l'illustre la Figure 8.

Choisir entre un modèle canté et un modèle colinéaire

Afin de montrer comment la PSN peut différencier une structure cantée d'une structure colinéaire, une structure tétragonale de vecteur de propagation $\boldsymbol{\tau}=0,0, \frac{1}{2}$ est utilisée comme exemple. Cette dernière possède 4 atomes magnétiques par maille : $\mathrm{A} 1$ en $(x, x, 0)$; $\mathrm{A} 2$ en $(-x, x, 0), \mathrm{A} 3$ en $(-x,-x, 0)$; $\mathrm{A} 4$ en $(x,-x, 0)$ avec $x \approx 0.2$, leurs spins étant dans le plan 001 .

Les configurations possibles du plan en $z=0$ sont présentées sur la Figure 9. L'expression des facteurs de structure magnétique pour les trois modèles est donnée dans le Table 1. A partir de ces expressions, on peut voir que :

- L'intensité moyenne diffractée par les deux domaines orthorhombiques est exactement la même que celle diffractée par la structure tétragonale.

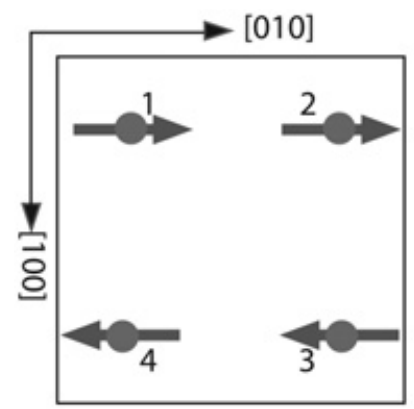

(a) $m m^{\prime} m^{\prime}$

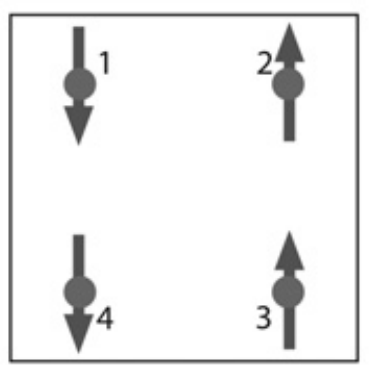

(b) m'mm'

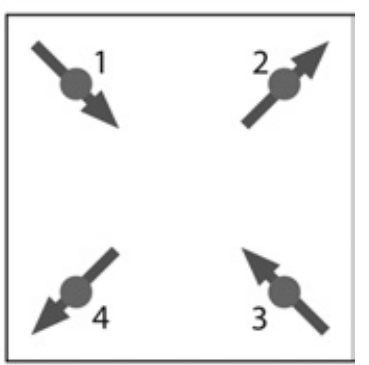

(c) $4 ' / \mathrm{m}^{\prime} \mathrm{mm}$

Figure 9. Plan à $\mathrm{z}=0$ pour les trois configurations de spins possibles dans une structure tétragonale avec $\tau=0,0, \frac{1}{2}$.

Table 1. Composantes des facteurs de structure magnétique $\mathbf{F}_{\mathbf{M}}(\mathbf{Q})$ pour les réflexions $h k l$.

\begin{tabular}{llll}
\hline Composante & Modèle (a) & Modèle (b) & Modèle (c) \\
\hline$M_{[100]}$ & 0 & $4 S s(h) c(k)$ & $2 \sqrt{2} S s(h) c(k)$ \\
$M_{[010]}$ & $4 S s(k) c(h)$ & 0 & $2 \sqrt{2} S s(k) c(h)$ \\
\hline Intensité & $(4 S s(k) c(h))^{2}$ & $(4 S s(h) c(k))^{2}$ & $8 S^{2}\left(s^{2}(h) c^{2}(k)+s^{2}(k) c^{2}(h)\right)$ \\
Moyenne des Domaines & $8 S^{2}\left(s^{2}(h) c^{2}(k)+s^{2}(k) c^{2}(h)\right)$ & \\
\hline
\end{tabular}

$c(h)=\cos 2 \pi h x ; s(h)=\sin 2 \pi h x c(k)=\cos 2 \pi k x ; s(k)=\sin 2 \pi k x$ 
- L'intensité moyenne diffractée par les deux domaines orthorhombiques est exactement la même que celle diffractée par la structure tétragonale.

- La structure tétragonale donne un unique $\mathbf{F}_{\mathbf{M}}(\mathbf{Q})$ pour chaque réflexion. La PSN permet de déterminer sa direction.

- Un mélange de domaines orthorhombiques induira une dépolarisation pour $\mathbf{P}$ perpendiculaire à $\mathbf{Q}$ car les facteurs de structure des deux domaines ne sont pas parallèles.

Table 2. Composantes des vecteurs d'interaction magnétique $\mathbf{M}_{\perp}(\mathbf{Q})$ pour les réflexions $0 k l$

\begin{tabular}{lllll}
\hline Composante & Modèle (a) & Modèle (b) & Modèle (c) & Notes \\
\hline$M_{\perp y}$ & 0 & $2 F^{\prime} \sin \alpha$ & $\sqrt{2} F^{\prime} \sin \alpha$ & $F=S \sin 2 \pi h x$ \\
$M_{\perp z}$ & $2 F$ & 0 & $\sqrt{2} F$ & $F^{\prime}=S \cos 2 \pi h x$ \\
\hline Intensité Magnétique & $4 F^{2}$ & $4 F^{\prime 2} \sin ^{2} \alpha$ & $2\left(F^{2}+F^{\prime 2} \sin ^{2} \alpha\right)$ & $I=F^{2}+F^{\prime 2} \sin ^{2} \alpha$ \\
\hline
\end{tabular}

Lors d'une expérience de PSN, afin de distinguer les modèles, le cristal doit être monté de sorte que les réflexions $h, 0, \frac{1}{2}+l$ soient dans le plan de diffraction : [010] parallèle à la polarisation $z$. Les composantes $M_{\perp y}, M_{\perp x}$ des vecteurs d'interaction magnétique pour les différents modèles sont donnés dans le Table 2 pour une réflexion $h 0 l$ dont le vecteur de diffusion est incliné d'un angle $\alpha$ par rapport à la direction [100]. Les matrices de polarisation correspondantes sont :

Modèle (a)

$P=\left(\begin{array}{rrr}-1 & 0 & 0 \\ 0 & -1 & 0 \\ 0 & 0 & 1\end{array}\right) \quad P=\left(\begin{array}{rrr}-1 & 0 & 0 \\ 0 & 1 & 0 \\ 0 & 0 & -1\end{array}\right)$

Modèle (c)

$$
P=\left(\begin{array}{ccl}
-1 & 0 & 0 \\
0 & -\left(F^{\prime 2}-F^{2} \sin ^{2} \alpha\right) / I & \left(2 F F^{\prime} \sin \alpha\right) / I \\
0 & \left(2 F F^{\prime} \sin \alpha\right) / I & \left(F^{\prime 2}-F^{2} \sin ^{2} \alpha\right) / I
\end{array}\right)
$$

La moyenne des domaines (a) et (b), égaux en volume, est

$$
P=\left(\begin{array}{cll}
-1 & 0 & 0 \\
0 & -\left(F^{\prime 2}-F^{2} \sin ^{2} \alpha\right) / I & 0 \\
0 & 0 & \left(F^{\prime 2}-F^{2} \sin ^{2} \alpha\right) / I
\end{array}\right)
$$

Seuls les termes non-diagonaux diffèrent. Ils peuvent être mesurés durant une expérience de PSN.

\subsection{Structures incommensurables}

La PSN a été utilisée avec succès pour la détermination précise de structures incommensurables telles que les hélices, les cycloïdes et les ondes de densité de spin. Elle permet également souvent de faire la différence entre ces types de structures.

Pour l'étude de structures incommensurables, il est important de bien réfléchir à l'orientation du cristal.

- Le vecteur de propagation doit être dans le plan de diffraction.

- Une des composantes du moment doit être perpendiculaire au plan de diffraction. Si les deux composantes du moment sont dans le plan de diffraction, alors $\mathbf{M}_{\perp}$ est parallèle à la polarisation $y$ pour toutes les réflexions accessibles. Lorsque $\tau \neq 0$, seule la direction de $\mathbf{M}_{\perp}$ est mesurée par PSN, mais pas son amplitude, si bien que la mesure de différentes réflexions ne donnera pas plus d'informations. 
- Si l'orientation est choisie de telle sorte que la composante du moment soit parallèle à la polarisation $z$ alors tout le potentiel de la PSN peut s'exercer.

La distribution des moments magnétiques dans une structure modulée sinusoïdale de vecteurs de réseau $\boldsymbol{l}$ et de vecteur de propagation $\tau$ peut être écrite par

$$
\mathbf{M}(\mathbf{r}+l)=\hat{\mathbf{p}} M_{p}(\mathbf{r}) \cos l \cdot \boldsymbol{\tau}+\hat{\mathbf{q}} M_{q}(\mathbf{r}) \sin l \cdot \boldsymbol{\tau}
$$

où $\hat{\mathbf{p}}$ et $\hat{\mathbf{q}}$ sont des vecteurs unitaires perpendiculaires.

- Lorsque $M_{\mathrm{p}}(\mathbf{r})$ ou $M_{\mathrm{q}}(\mathbf{r})$ est nul, l'équation décrit une onde de densité de spin.

- Lorsque $\boldsymbol{\tau}$ est dans le plan de $\hat{\mathbf{p}}$ et $\hat{\mathbf{q}}$, cette équation 4.4 décrit une cycloïde.

- Lorsque les deux sont perpendiculaires à $\tau$, elle décrit une hélice droite.

La diffraction magnétique d'une telle structure modulée est donnée par

$$
\begin{aligned}
\mathbf{M}_{\perp}(\mathbf{Q}) & =\left(\mathbf{p}_{\perp} M_{\mathrm{p}}(\mathbf{Q})-\imath \mathbf{q}_{\perp} M_{\mathrm{q}}(\mathbf{Q})\right) \delta(\mathbf{g}+\boldsymbol{\tau}-\mathbf{Q}) \\
& +\left(\mathbf{p}_{\perp} M_{\mathrm{p}}(\mathbf{Q})+\imath \mathbf{q}_{\perp} M_{\mathrm{q}}(\mathbf{Q})\right) \delta(\mathbf{g}-\boldsymbol{\tau}-\mathbf{Q})
\end{aligned}
$$

où $\mathbf{p}_{\perp}$ et $\mathbf{q}_{\perp}$ sont les composantes de $\hat{\mathbf{p}}$ et $\hat{\mathbf{q}}$ perpendiculaires à $\mathbf{Q}$.

$$
M_{\mathrm{p}, \mathrm{q}}(\mathbf{Q})=\int_{\text {unite de cellule }} M_{\mathrm{p}, \mathrm{q}}(\mathbf{r}) \exp -(\mathbf{Q} \cdot \mathbf{r}) d r^{3}
$$

sont les facteurs de structure de la cellule unité pour les deux distributions de moments magnétiques perpendiculaires.

- Une structure avec un vecteur de propagation $\boldsymbol{\tau}$ donne des réflexions à la fois en $\mathbf{g}+\boldsymbol{\tau}$ et $\mathbf{g}-\boldsymbol{\tau}$.

- Pour des structures pour lesquelles les $M_{\mathrm{p}, \mathrm{q}}(\mathbf{Q})$ sont réels, ie les distributions d'aimantation ellesmêmes sont centro-symétriques, $\mathbf{M}_{\perp}(\mathbf{Q}) \boldsymbol{\tau}=\mathbf{M}_{\perp}^{*}(\mathbf{Q})-\boldsymbol{\tau}$

- Le terme de la matrice de polarisation qui crée de la polarisation selon $x$ :

$J_{i j}=2 \Re\left(M_{\mathrm{p}}(\mathbf{Q}) M_{\mathbf{q}}(\mathbf{Q})\right)\left|\mathbf{p}_{\perp} \times \mathbf{q}_{\perp}\right|$ est fini si ni $\mathbf{p}_{\perp}$, ni $\mathbf{q}_{\perp}$ ne sont nuls ou si le facteur de structure $M_{\mathrm{p}, \mathrm{q}}(\mathbf{Q})$ est égal à zéro.

Un exemple va permettre de montrer comment les mesures de PSN peuvent déterminer une structure quand les $M_{p, q}(\mathbf{Q})$ sont réels. Le cristal doit être aligné avec $\boldsymbol{\tau}$ dans le plan de diffraction. On suppose pour simplifier que $\hat{\mathbf{p}}$ est perpendiculaire au plan de diffraction de sorte que $\hat{\mathbf{q}}$ soit dans ce plan. Pour les différentes réflexions du plan de diffraction, $\mathbf{p}_{\perp} \| z$ est constant, tandis que $\mathbf{q}_{\perp} \| y$ varie entre 0 lorsque $\hat{\mathbf{q}} \| \mathbf{Q}$ et un maximum quand $\hat{\mathbf{q}} \perp \mathbf{Q}$.

Les matrices de polarisation qui doivent être obtenues sont :

$$
\begin{aligned}
& \mathbf{P}=\left(\begin{array}{rrr}
-1 & 0 & 0 \\
B & A & 0 \\
B & 0 & -A
\end{array}\right) \quad \text { for } \mathbf{Q} \| \hat{\mathbf{q}} \quad \text { avec } \quad A=\frac{M_{\mathrm{p}}(\mathbf{Q})^{2}-M_{\mathrm{q}}(\mathbf{Q})^{2}}{M_{\mathrm{p}}(\mathbf{Q})^{2}+M_{\mathrm{q}}(\mathbf{Q})^{2}} \\
& \mathbf{P}=\left(\begin{array}{ccc}
-1 & 0 & 0 \\
0 & -1 & 0 \\
0 & 0 & 1
\end{array}\right) \quad \text { for } \mathbf{Q} \perp \hat{\mathbf{q}} \quad \text { et } \quad B=\frac{2 M_{\mathrm{p}}(\mathbf{Q}) M_{\mathrm{q}}(\mathbf{Q})}{M_{\mathrm{p}}(\mathbf{Q})^{2}+M_{\mathrm{q}}(\mathbf{Q})^{2}} \\
& \mathbf{P}=\left(\begin{array}{rrr}
-1 & 0 & 0 \\
-B & A & 0 \\
-B & 0 & -A
\end{array}\right) \quad \text { for } \mathbf{Q} \| \hat{\mathbf{q}} \quad \text { pour l'autre domaine de chiralité }
\end{aligned}
$$


Les points à souligner sont :

- $P x x=-1$ dans les trois cas ; en fait il doit toujours en être ainsi lorsque $\tau \neq 0$.

- Pour $\mathbf{P} \perp \mathbf{Q}$, il n'y a pas de composante $y$ de $\mathbf{M}_{\perp}(\mathbf{Q})$ et donc le comportement est le même que pour une structure colinéaire avec $\mathbf{M}_{\perp}(\mathbf{Q}) \| \mathbf{z}$.

- Pour $\mathbf{P} \| \mathbf{Q}$, les composantes $P_{y y}$ et $P_{z z}$ donnent l'éllipticité de l'hélice. Elles seront nulles si l'enveloppe est circulaire. L'intensité diffractée par le domaine pour lequel $B$ est positif sera plus grande que celle de celui pour lequel $B$ est négatif.

- Les composantes $P_{y x}$ et $P_{z x}$ sont de signes opposés pour les deux domaines de chiralité et, s'ils sont tout deux peuplés de façon égale, elles se moyenneront à zéro donnant lieu à une matrice de polarisation diagonale.

La structure incommensurable de l'oxide de cuivre

A sa température de Néel, $230 \mathrm{~K}, \mathrm{CuO}$ s'ordonne magnétiquement dans une structure incommensurable, $\tau=0.506,0,-0.483$, qui reste invariable à plus basse température jusqu'à une transition dans la phase commensurable à $213 \mathrm{~K}$. Les raies magnétiques systématiquement absentes suivent les mêmes lois que dans la phase basse température (section 4.2.). Ceci suggère que les couplages entre les moments dans les deux phases sont presque les mêmes. Des mesures d'intensités intégrées ne permettaient pas de distinguer clairement entre les différents modèles possibles de la modulation [3]. Les mesures en PSN de quelques réflexions $h 0 l \pm \tau$ ont permis de résoudre le problème [4].

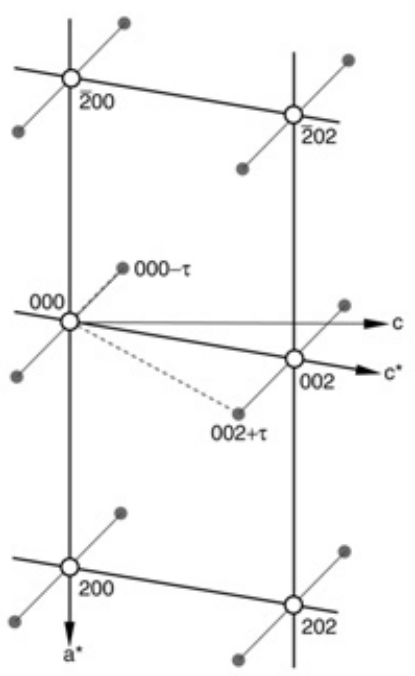

(a)

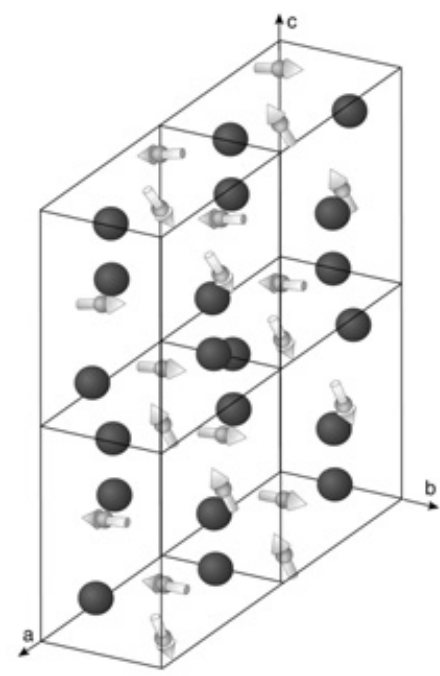

(b)

Figure 10. (a) Plan $h 0 l$ du réseau réciproque de $\mathrm{CuO}$, montrant les positions des réflexions magnétiques de la phase incommensurable. (b) Structure magnétique incommensurable de $\mathrm{CuO}$.

Les vecteurs de diffusion pour les réflections $002+\tau$ et $000-\tau$ sont presque perpendiculaires. La matrice de polarisation pour la $002+\tau$ était similaire à celles mesurées dans la phase commensurable. Ainsi, pour la $002+\tau, \mathbf{M}_{\perp}$ est parallèle à [010]. La matrice obtenue pour la $000-\boldsymbol{\tau}$ était en revanche très différente.

$$
P(000-\tau)=\left(\begin{array}{lll}
-1.00 & 0.00 & -0.04 \\
-0.08 & -0.07 & 0.00 \\
-0.08 & 0.00 & 0.06
\end{array}\right)
$$


- La totalité de la polarisation n'est transmise que dans la direction $x$.

- Les petites valeurs de $\mathrm{P}_{x y}$ et $\mathrm{P}_{x z}$ sont dues à l'annulation des éléments non-diagonaux par les domaines de chiralités opposées.

- Les petites valeurs de $\mathrm{P}_{y y}$ et $\mathrm{P}_{z z}$ montrent que pour cette réflexion $M_{\perp p}$ et $M_{\perp q}$ sont presque égaux.

Ces résultats sont cohérents uniquement avec la structure en hélice présentée sur la Figure 10(b) dans laquelle les spins tournent dans un plan contenant l'axe $\mathbf{b}$ et la normale à $002+\tau$.

\section{Structure magnétique de UPtGe}

Une transition magnétique à $51 \mathrm{~K}$ en une structure cycloïdale vec un vecteur de propagation $\boldsymbol{\tau}=0,0.554,0$ et les spins des atomes $\mathrm{U}$ tournant dans le plan $\mathbf{b}-\mathbf{c}$ avait été proposée pour le composé intermétallique orthorhombique UPtGe comme l'illustre la Figure 11(a) [6]. La possibilité d'une telle structure a été remise en question étant donnée l'anisotropie magnétique énorme de l'uranium observée dans les autres composés UTX.

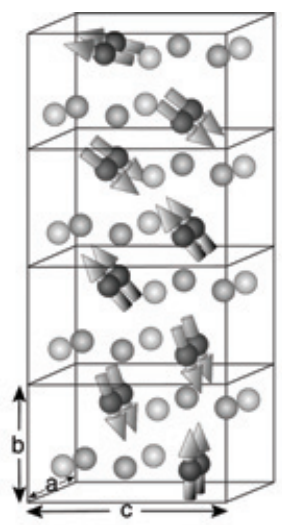

(a)

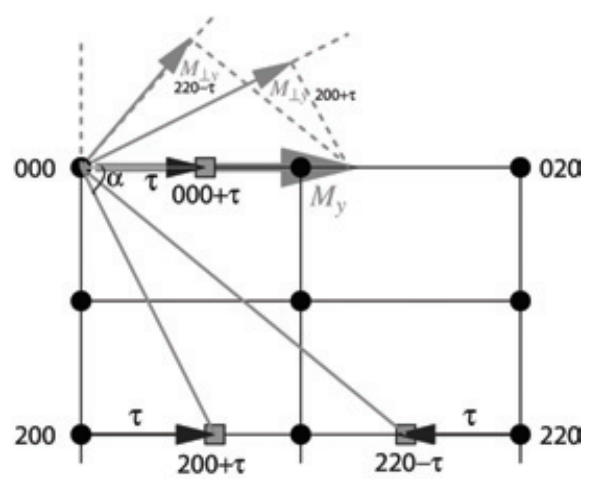

(b)

Figure 11. (a) Structure magnétique cycloïdale de UPtGe et (b) plan $h k 0$ du réseau réciproque correspondant.

La structure proposée a été vérifiée, en mesurant les matrices de polarisation des réflexions du plan $h k 0$ montrées sur la Figure 11(b), ce qui a conduit aux résultats suivants :

$$
\begin{aligned}
\mathbf{P}(000+\tau)= & \left(\begin{array}{ccc}
-0.98 & 0.00 & 0.00 \\
0.00 & -0.99 & 0.00 \\
0.00 & 0.00 & 1.00
\end{array}\right) \\
& \text { montre que } \mathbf{M}_{\perp} \| z \\
\mathbf{P}(200+\tau)= & \left(\begin{array}{lll}
0.50 & 0.00 & 0.00 \\
1.02 & 0.00 & 0.00 \\
1.02 & 0.02 & 0.04
\end{array}\right) \\
& \text { donne } \mathbf{P}^{\prime} \|-x \text { quelque soit } \mathbf{P}
\end{aligned}
$$
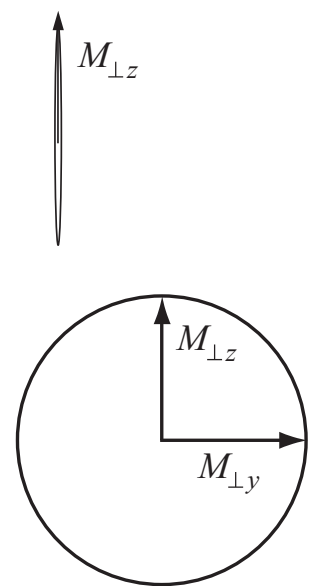
Avec $\mathbf{P} \| \mathbf{x}$, l'intensité diffractée est très petite et $\mathbf{P}_{x x}$ n'est pas déterminé de façon précise.

$$
\begin{aligned}
& \mathbf{P}(220-\tau)=\left(\begin{array}{ccc}
-0.98 & 0.05 & -0.05 \\
-0.98 & -0.10 & -0.02 \\
-0.98 & 0.01 & 0.17
\end{array}\right) \\
& \mathbf{P}^{\prime} \|-x \text { avec } \mathrm{P}_{\text {zz }} \text { petit }
\end{aligned}
$$

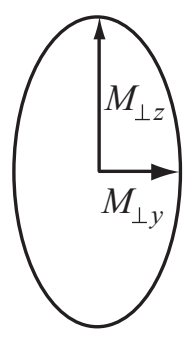

Ces résultats sont caractéristiques d'une structure cycloïdale pratiquement mono-domaine dans laquelle le domaine peuplé est celui où $\tau \|-x$ [7].

L'éllipticité de l'enveloppe, $\epsilon=\frac{M_{[010]}}{M_{[001]}}=\frac{\text { axe majeur }}{\text { axe mineur }}$, et l'orientation de l'axe majeur de la cycloïde ont été obtenues en affinant

$$
\mathbf{P}_{y y}(\mathbf{Q})=-\mathbf{P}_{z z}(\mathbf{Q})= \pm \frac{\epsilon^{2} \sin ^{2} \phi_{\mathbf{Q}}-1}{\epsilon^{2} \sin ^{2} \phi_{\mathbf{Q}}+1}
$$

pour un jeu de réflexions magnétiques ; $\phi_{\mathbf{Q}}$ est l'angle entre l'axe majeur et $\mathbf{Q}$.

\subsection{Structures magnétiques avec un vecteur de propagation nul}

Comme il a été dit dans la section 2.3, lorsque le vecteur de propagation magnétique est nul, les diffractions magnétique et nucléaire peuvent avoir lieu sur les mêmes réflexions. Les termes

$$
J_{n i}=2 \Im\left(F_{N}(\mathbf{Q}) M_{\perp i}^{*}(\mathbf{Q})\right) \quad \text { et } \quad R_{n i}=2 \Re\left(F_{N}(\mathbf{Q}) M_{\perp i}^{*}(\mathbf{Q})\right)
$$

dans la matrice de polarisation (équations 2.13 et 2.14) peuvent être non-nuls. Une valeur de $R_{n i}$ finie donne lieu à une section efficace dépendante de la polarisation. Le faisceau diffracté est polarisé parallèlement à $\mathbf{M}_{\perp}$. Ceci se produit lorsque le déphasage entre $\mathbf{M}_{\perp}(\mathbf{Q})$ et $F_{N}(\mathbf{Q})$ est différent de $(2 n+1) \pi$. Il s'agit du terme utilisé pour déterminer les facteurs de structure magnétique à partir des rapports de flipping. Une valeur de $J_{n i}$ finie donne lieu à une rotation de la polarisation diffractée vers la direction perpendicualire à la fois à $\mathbf{M}_{\perp}$ et $\mathbf{P}$. Ceci se produit quand le déphasage entre $\mathbf{M}_{\perp}(\mathbf{Q})$ et $F_{N}(\mathbf{Q})$ est différent de $2 n \pi$.

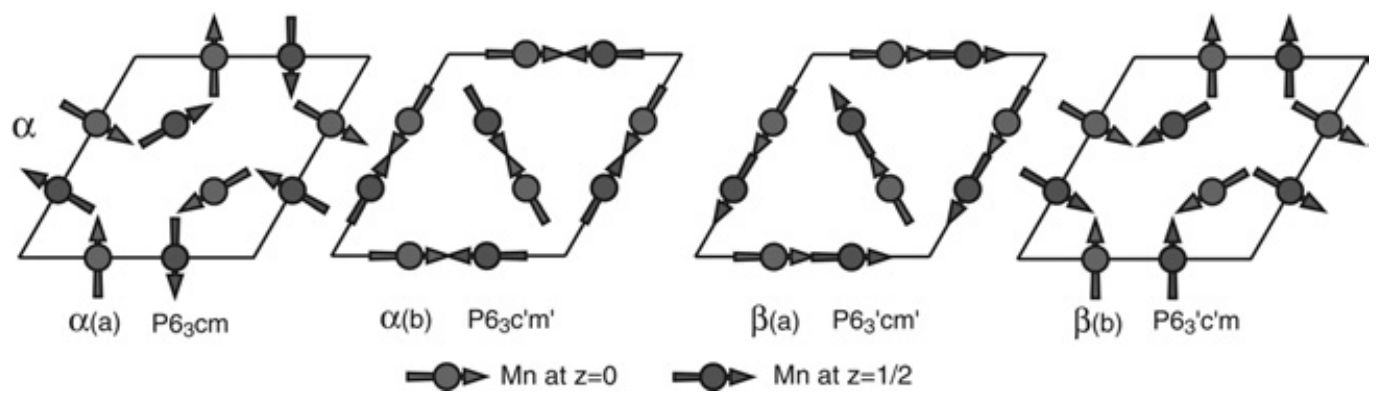

Figure 12. Projections selon l'axe [001] montrant les configurations de spins possibles pour les maganites de terre-rare. 


\section{Résolution d'homométrie dans les manganites RE}

Les manganites $\mathrm{RMnO}_{3}$ d'ions terre-rare les plus petits $(\mathrm{R}=\mathrm{Y}, \mathrm{Ho}, \mathrm{Er}, \mathrm{Tm}, \mathrm{Yb}, \mathrm{Lu})$ cristallisent dans une structure hexagonale [8]. Les manganèses occupent les sites $(x, 0,0)$ avec $x \approx 1 / 3$. Ils s'ordonnent magnétiquement aux alentours de $80 \mathrm{~K}$ avec leurs moments dans le plan (001) formant un réseau triangulaire. D'autres arrangements de symétries différentes, qui ont été proposés, sont représentés sur la Figure 12.

Table 3. Contributions des moments des manganèses aux composantes des vecteurs d'interaction magnétique des réflexions $h 0 l$ pour différents modèles d'ordre magnétique des maganites de terre-rare.

\begin{tabular}{llll}
\hline Modèle & Parité & \multicolumn{1}{c}{$M_{\perp y}$} & $M_{\perp z}$ \\
\hline \multirow{2}{*}{$\alpha(a)$} & $l$ impair & $(G-1) \cos \alpha$ & 0 \\
& $l$ pair & 0 & $\imath \sqrt{3} G^{\prime}$ \\
\multirow{2}{*}{$\beta(a)$} & $l$ impair & $\imath \sqrt{3} G^{\prime} \cos \alpha$ & 0 \\
& $l$ pair & 0 & $1-G$ \\
\hline \multirow{2}{*}{$\alpha(b)$} & $l$ impair & 0 & $1-G$ \\
& $l$ pair & $\imath \sqrt{3} G^{\prime} \cos \alpha$ & 0 \\
\multirow{2}{*}{$\beta(b)$} & $l$ impair & 0 & $\imath \sqrt{3} G^{\prime}$ \\
& $l$ pair & $(G-1) \cos \alpha$ & 0 \\
\hline
\end{tabular}

$\begin{aligned} G & =\cos 2 \pi h x_{\mathrm{Mn}} \\ G^{\prime} & =\sin 2 \pi h x_{\mathrm{Mn}} \\ \alpha & =\tan ^{-1} \sqrt{3} l a / 2 h c\end{aligned}$

Lorsque $x_{\mathrm{Mn}}=\frac{1}{3}, \quad|1-G|^{2}=3\left|G^{\prime}\right|^{2}$ quelque soit $h$

Les structures magnétiques n'ont jamais été complètement résolues. Les structures a et b de la Figure 12 peuvent être différenciées par des mesures d'intensités, mais si $x=1 / 3$, les intensités magnétiques pour les paires $\alpha$ et $\beta$ sont exactement les mêmes [9]. Comme $x$ ne vaut pas exactement 1/3, cette homométrie n'est pas exacte mais les intensités ne sont pas suffisamment différentes pour permettre de distinguer entre les structures de type $\alpha$ et de type $\beta$. Dans $\mathrm{YMnO}_{3}$, les mesures d'intensités montrent que la structure est de type b. Dans $\mathrm{HoMnO}_{3}$, une transition d'une structure de type a vers une structure de type $\mathrm{b}$ a lieu aux alentours de $37 \mathrm{~K}$.

Le Table 3 montre que bien que les intensités des réflexions magnétiques pour les modèles $\alpha$ et $\beta$ soient presques les mêmes, leurs phases sont très différentes et donc les termes d'interférences nucléairemagnétique diffèrent largement. La PSN offre ainsi la possibilité d'une solution directe à l'homométrie par l'exploitation de la sensibilité en direction et en phase de l'analyse de polarisation.

Puisque la structure est non-centrosymétrique, les facteurs de structure nucléaire sont complexes. Ils peuvent être écris en fonction d'un module $N$ et d'une phase $\phi$ comme

$$
F_{N}(\mathbf{Q})=N(\mathbf{Q}) \cos \phi+\imath N(\mathbf{Q}) \sin \phi
$$

Le déséquilibre entre les populations de domaines à $180^{\circ}$ dans le cristal peut être défini par une fraction de domaines $\eta$

$$
\eta=\frac{v_{1}-v_{2}}{v_{1}+v_{2}}
$$

où $v_{1}$ et $v_{2}$ sont les volumes du cristal appartenant à chacun des deux domaines. Les matrices de polarisation qui seraient mesurées pour les réflexions $h 0 l$ sont données en fonction de $\eta$ et de facteurs de structures nucléaire et magnétique dans le table 4 . Tant que $\eta \neq 0$, la distinction entre les deux modèles est nette. Malheureusement, il semble difficile de déséquilibrer les populations de domaines et donc, jusqu'à présent, cette méthode n'a pas permis de résoudre les structures. 
Table 4. Matrices de polarisation des réflexions $h 0 l$ calculées pour les différents modèles de structures magnétiques de $\mathrm{YMnO}_{3}$.

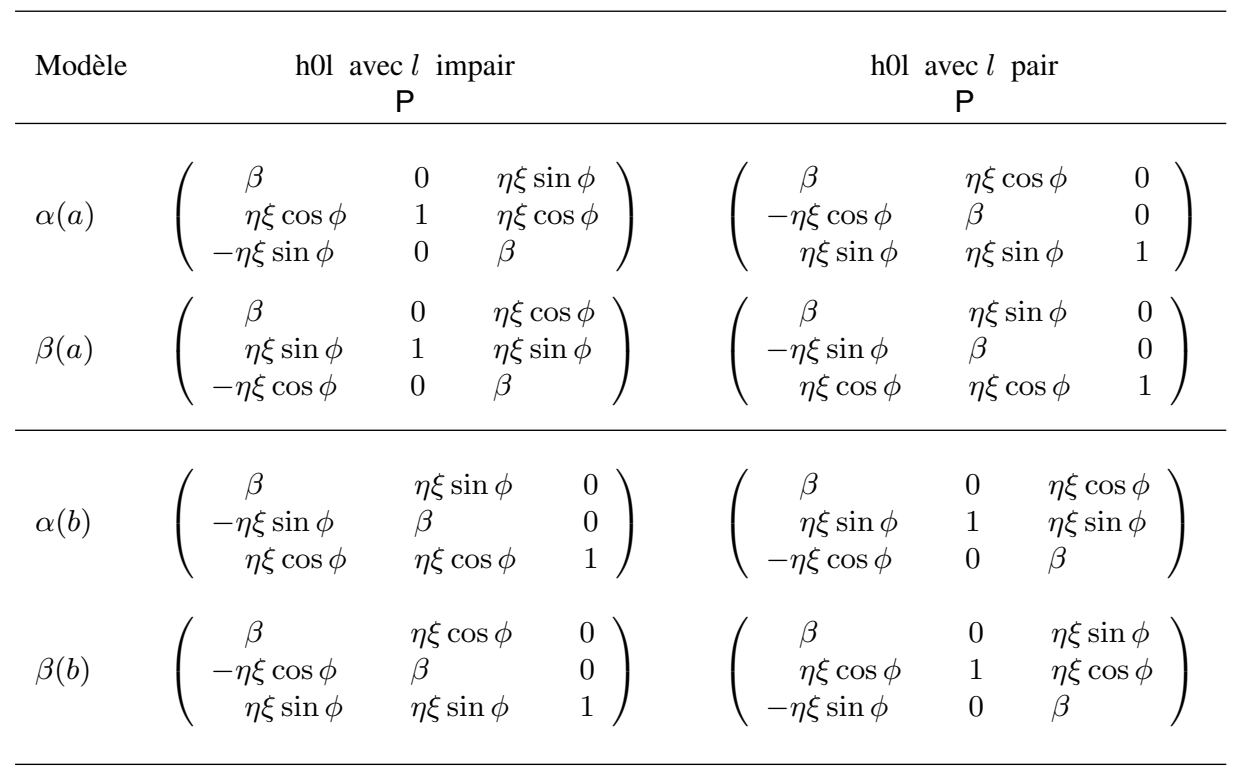

$\beta=\left(1-\gamma^{2}\right) /\left(1+\gamma^{2}\right) ; \xi=q \gamma /\left(1+\gamma^{2}\right)$

$\gamma=\left|\mathbf{M}_{\perp}\right| / N ; F_{N}(\mathbf{Q})=N \cos \phi+\imath N \sin \phi$

$q$ vaut +1 si $\mathbf{M}_{\perp}$ estparallleyouz, et-1 s'ilestantiparallle.

\section{Cristaux magnéto-électriques}

La propriété de magnéto-électricité dans les cristaux centrosymétriques n'apparaît que dans ceux qui présentent des structures antiferromagnétiques avec un vecteur de propagation nul et dans lesquelles le centre de symétrie est combiné avec le renversement du temps. Ces conditions sont justement celles requises pour que les $J_{n i}$ dans les équations 2.13 et 2.14 soient finis, donnant lieu à des termes nondiagonaux $P_{x z}, P_{z x}$ dans la matrice de polarisation. L'origine peut être choisie de sorte que $F_{N}$ soit réel, auquel cas $\mathbf{M}_{\perp}$ est imaginaire pur et $J_{n i}=2 F_{N} M_{\perp i}$.

Bien que les dépendances en température des susceptibilités magnéto-électriques (ME) soient uniques pour chaque matériaux, leurs amplitudes et même leurs signes dépendent des échantillons. Cette dépendance est due à l'existence de domaines à $180^{\circ}$ qui ont des effets ME opposés. La susceptibilité ME mesurée $\chi_{o b s}$ est reliée à la susceptibilité intrinsèque $\chi_{0}$ par

$$
\chi_{o b s}=\eta \chi_{0}
$$

où $\eta$ est la fraction de domaine définie dans l'équation 4.6. La PSN permet, pour la première fois, d'obtenir la susceptibilité ME intrinsèque puisqu'elle rend possible la détermination de la fraction de domaine $\eta$.

Pour un cristal centro-symétrique ME avec une fraction de domaines $\eta$ et les moments dans le plan $\mathbf{x}-\mathbf{y}$, la matrice de polarisation peut être simplifiée en

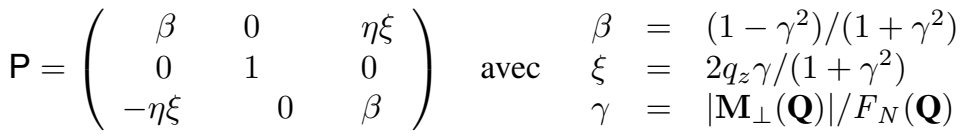

$q_{z}$ vaut +1 si $\mathbf{F}_{\mathbf{M}}(\mathbf{Q})$ est parallèle à $\mathbf{z}$, et -1 si $\mathbf{F}_{\mathbf{M}}(\mathbf{Q})$ et $\mathbf{z}$ sont antiparallèles. 
- La mesure de la matrice de polarisation permet de déterminer à la fois $\eta$ et $\gamma$.

- Les directions absolues de rotation des spins des neutrons lorsque $\eta \neq 0$ déterminent la configuration magnétique du domaine le plus peuplé, ce qui permet d'étudier les effets des champs électrique et magnétique sur les populations de domaines.

- Les résultats peuvent permettre de comprendre les mécanismes fondamentaux produisant l'effet ME.
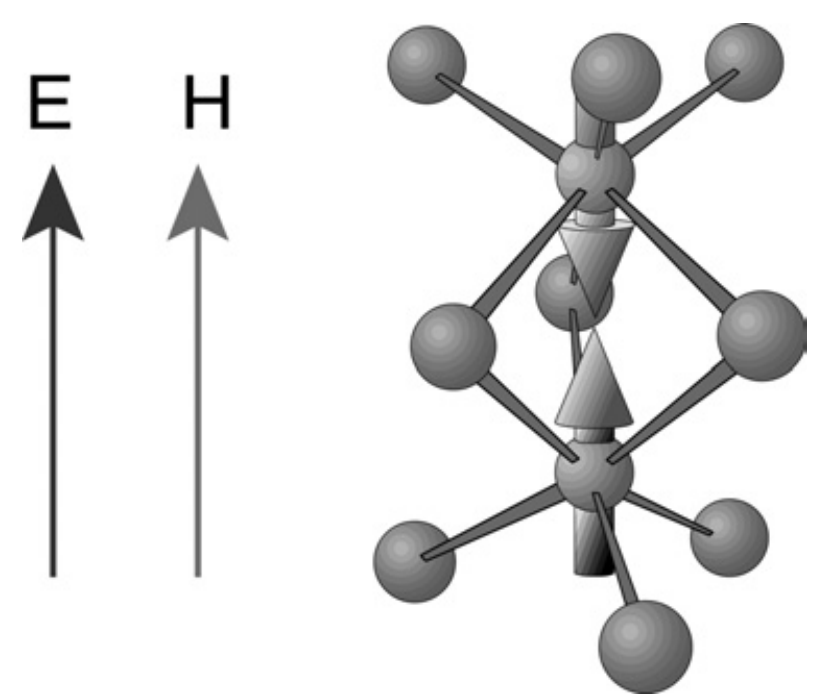

Figure 13. Paire d'octaèdres de coordination à face commune entourant les ions $\mathrm{Cr}^{3+}$ dans $\mathrm{Cr}_{2} \mathrm{O}_{3}$, montrant la direction relative des spins stabilisée par des champs électrique et magnétique parallèles.

$\mathrm{Cr}_{2} \mathrm{O}_{3}$ est peut-être le plus connu des matériaux ME. Les ions $\mathrm{Cr}^{3+}$ sont au centre d'un octaèdre d'oxygènes et la structure est composée de paires d'octahèdres partageant une face commune. Ces doubles octahèdres sont liés aux autres par le partage de leurs sommets libres. La PSN a montré que les champs électrique et magnétique, appliqués parallèlement entre eux et à l'axe, lors du passage de la transition de Néel, stabilisent le domaine dans lequel les moments pointent vers la face commune de leurs octaèdres d'oxygènes de coordination, comme c'est indiqué sur la Figure 13.

\section{LA PSN POUR UNE DÉTERMINATION PRÉCISE DES FACTEURS DE STRUCTURE MAGNÉTIQUE}

\subsection{Les facteurs de structure magnétique à partir de la rotation de la polarisation}

Les matrices de polarisation données par l'équation 4.8, pour les réflexions de cristaux dans lesquels les diffractions magnétique et nucléaire sont en quadrature, ne dépendent que du rapport $\gamma$ entre les facteurs de structures magnétique et nucléaire et du déséquilibre $\eta$ de populations des deux domaines à $180^{\circ}$ Cette propriété peut être exploitée afin d'obtenir des valeurs précises des facteurs de structure magnétique et donc du facteur de forme antiferromagnétique [10]. La matrice de polarisation permet d'obtenir deux estimations indépendantes de $\gamma$, provenant de chacune des équations 5.1(a) et (b). (a) n'est utile que s'il y a un déséquilibre $\eta$ de populations des domaines à $180^{\circ}$.
(a) $\mathbf{P}_{x z}=-\mathbf{P}_{z x}=\eta \xi=\frac{\eta q_{y} \gamma}{1+\gamma^{2}}$
(b) $\mathrm{P}_{x x}=\mathrm{P}_{z z}=\beta=\frac{1-\gamma^{2}}{1+\gamma^{2}}$ 


\subsection{Considérations experimentales}

La précision avec laquelle $\gamma$ peut être déterminé dépend de l'erreur statistique faite sur la détermination de P. Dans ce type de structure, la section efficace est indépendante de la direction de la polarisation. Le taux de comptage sommé sur les deux états de polarisation acceptés par le détecteur est constant et indépendant des directions de polarisations incidente ou diffractée. La polarisation mesurée par l'analyseur est donnée par :

$$
P=\left(I^{+}-I^{-}\right) /\left(I^{+}+I^{-}\right)
$$

où $I^{+}$et $I^{-}$sont les taux de comptages dans les deux canaux du détecteur.

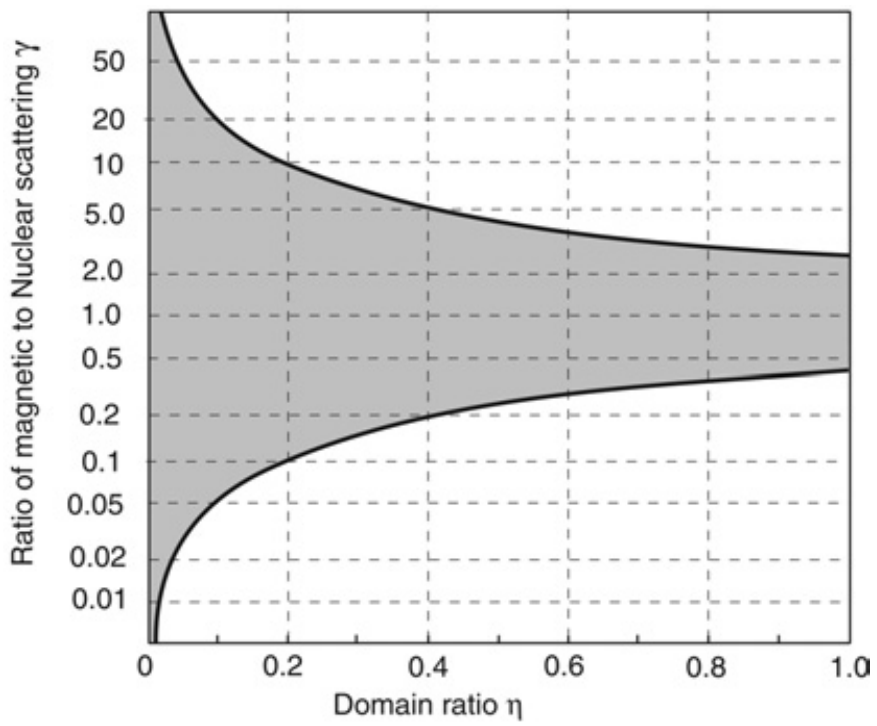

Figure 14. Schéma de l'espace $\gamma-\eta$. La région grisée est celle dans laquelle l'équations 5.1(a) donne une estimation plus précise de $\gamma$ que l'équations 5.1(b). L'axe $\gamma$ est tracé en échelle logarithmique.

- La variance, due à la statistique de comptage, de la mesure d'une composante de la polarisation est

$$
V_{P}=\frac{\left(1-P^{2}\right)^{2}}{4}\left(\frac{1}{N^{+}}+\frac{1}{N^{-}}\right)
$$

où $N^{+}$et $N^{-}$sont les comptages enregistrés dans chaque canal.

- La variance est minimisée en divisant le temps de mesure disponible dans le rapport $t^{+} / t^{-}=(1-P) /(1+P)$.

- Avec cette division, si le nombre total de neutrons compté est $N$, la variance devient

$$
V_{P}=\left(1-P^{2}\right) / N
$$

- Les variances des valeurs de $\gamma$ obtenues à partir des équations 5.1(a) et (b) sont
(a) $V_{\gamma}=\frac{\left(1+\gamma^{2}\right)^{4}}{16 \gamma^{2}} V_{P}$
(b) $V_{\gamma}=\frac{\left(1+\gamma^{2}\right)^{4}}{4 \eta^{2}\left(1-\gamma^{2}\right)^{2}} V_{P}$ 
La Figure 14 montre les zones dans le plan $\gamma-\eta$ où l'une ou l'autre, de l'équation 5.1(a) ou de l'équation 5.(b), donne la valeur la plus précise de $\gamma$. Si $\eta$ est petit (domaines presque égaux) ou si $\gamma$ est proche de l'unité, la meilleure estimation de $\gamma$ sera obtenue à partir de l'équation 5.1(b). Pour les valeurs très petites ou très grandes de $\gamma$, l'équation 5.1(a) donnera une meilleure estimation, tant que $\eta$ ne sera pas trop petit.

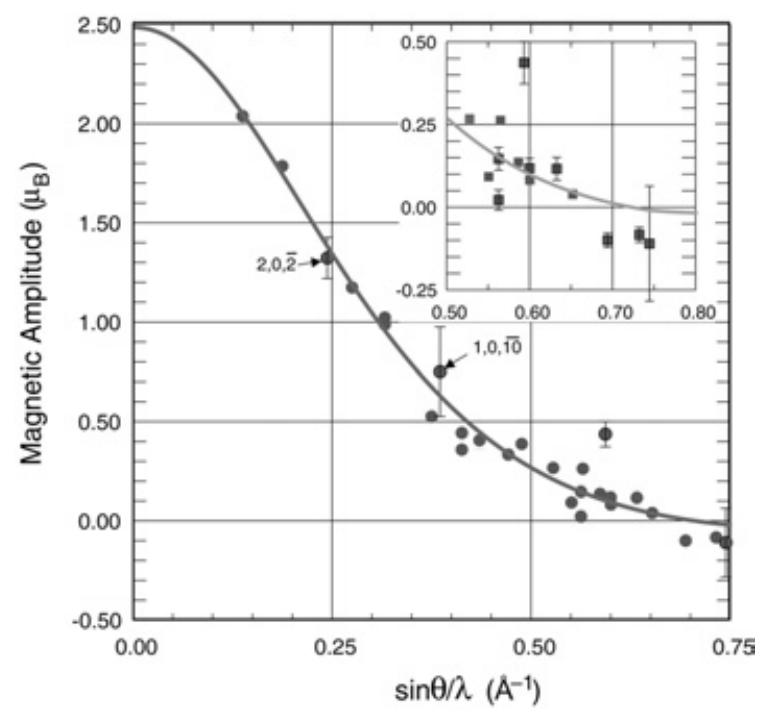

Figure 15. Valeurs expérimentales du facteur de forme magnétique mesurées à partir des réflexions de Bragg $h 0 l$ de $\mathrm{Cr}_{2} \mathrm{O}_{3}$. La courbe continue est le facteur de forme calculé en ne considérant que le spin de l'ion-libre $\mathrm{Cr}^{3+}$, normalisé à la valeur expérimentale de la réflexion à plus bas angle (10.2).

\subsection{Détermination du facteur de forme de $\mathbf{C r}^{3+}$ dans $\mathrm{Cr}_{2} \mathrm{O}_{3}$}

Les polarisations diffractées par un jeu de réflexions $h 0 l$ de $\mathrm{Cr}_{2} \mathrm{O}_{3}$ ont été mesurées dans un cristal présentant plusieurs états de populations de domaines différentes générés lors du refroidissement sous champ. Les points du facteur de forme $\mathrm{du}^{3} \mathrm{r}^{3+}$ ont été obtenus en multipliant les valeurs expérimentales de $\gamma$ par le rapport entre le facteur de structure nucléaire et le facteur de structure géométrique des sites de Cr. Le résultat est présenté sur la Figure 15. Pour la plupart des réflexions, une très bonne précision a été obtenue, à l'exception des réflexions $2,0, \overline{2}$ et $1,0, \overline{10}$. Le facteur de structure nucléaire de la $2,0, \overline{2}$ étant très petit, $\gamma>>1$. Pour la $1,0, \overline{10}$, c'est en revanche le facteur de structure géométrique pour les sites de $\mathrm{Cr}$ qui est petit de sorte que cette réflexion est insensible au facteur de forme. Les données ont également été utilisées pour faire une reconstruction par maximum d'entropie de la distribution d'aimantation antiferromagnétique. La Figure 16 montre la différence entre la distribution déterminée expérimentalement et celle calculée pour un arrangement antiferromagnétique d'ions $\mathrm{Cr}^{3+}$ de symétrie $t_{2 g}$. On peut remarquer qu'un gradient d'aimantation existe aux positions des $\mathrm{Cr}^{3+}$. Ceci peut être la signature d'un effet ME.

\section{RÉSUMÉ}

- La polarisation des neutrons diffractés est particulièrement sensible à la direction des moments magnétiques.

- Pour un système pur (sans domaines), la PSN permet de déterminer la direction des vecteurs d'interaction magnétique. 


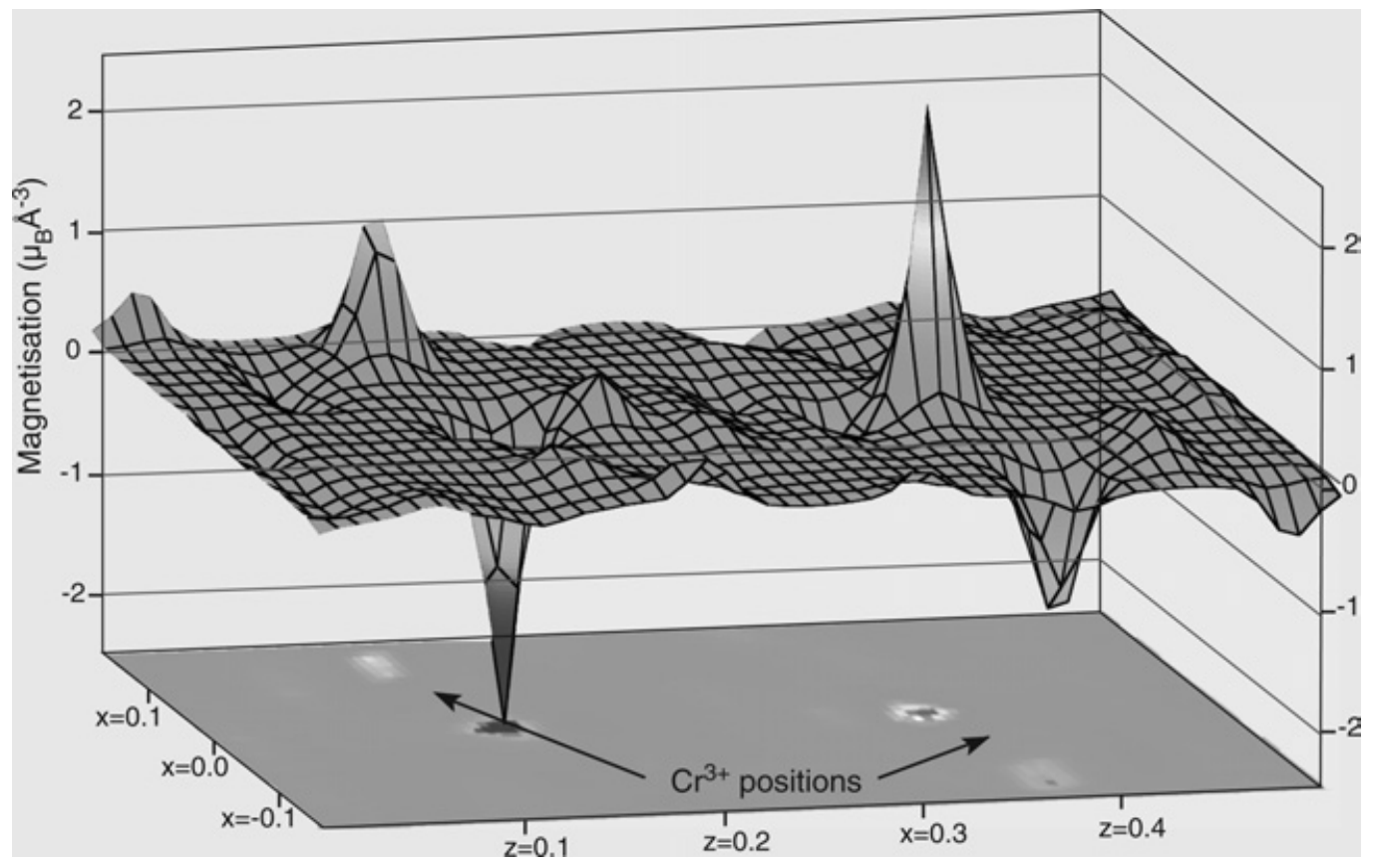

Figure 16. Reconstruction par maximum d'entropie de la différence entre la distribution d'aimantation expérimentale dans $\mathrm{Cr}_{2} \mathrm{O}_{3}$ et celle calculée pour un arrangement antiferromagnétique d'ions $\mathrm{Cr}^{3+}$ de symétrie $t_{2 g}$.

- La PSN permet de distinguer une véritable dépolarisation, due à la présence de domaines, d'une rotation de la polarisation par diffraction.

- L'observation d'une dépolarisation, quelle qu'elle soit, permettra de caractériser les types de domaines présents.

- Une rotation de la polarisation vers le vecteur de diffusion, ou s'en éloignant, indique un degré de chiralité dans la structure magnétique elle-même ou dans son interaction avec la structure nucléaire.

- La PSN ne peut être utilisée seule pour la détermination de structures magnétiques de vecteurs de propagation non-nuls.

- La mesure des matrices de polarisation d'un très petit nombre de réflexions magnétiques est normalement suffisante pour déterminer les directions des moments.

- Lorsque la relation de phase entre les structures magnétique et nucléaire peut être controllée, la PSN peut être utilisée afin d'obtenir des facteurs de structure magnétiques pr écis.

\section{Références}

[1] Blume, M., Phys. Rev. 1301670 (1963).

[2] Maleev, S.V., Bar'yaktar V.G. and Suris P.A., Sov. Phys. - Solid State 42533 (1963).

[3] Forsyth, J.B., Brown, P.J. and Wanklyn, B.M., J. Phys. C 212917 (1988).

[4] Brown, P.J., et al., J. Phys. Condens. Matter 34281 (1991).

[5] Brown, P.J., Forsyth, J.B. and Tasset, F., J.Phys. Condens. Matter 10663 (1998).

[6] Robinson, R., et al., Phys. Rev. B 476138 (1993).

[7] Mannix, D., et al., Phys. Rev. B 623810 (2000). 
[8] Yakel, H.L., Koehler, W.C., Bertaut, E.F. and Forrat, E.F., 169571963 (Acta Cryst.).

[9] Bacon, G.E., Neutron Diffraction OUP Oxford (1975)pp 488-495.

[10] Brown, P.J., Forsyth, J.B. and Tasset, F., Physica B 267-268 215 (1999).

[11] Brown, P.J., Forsyth, J.B., Lelièvre-Berna, E. and Tasset, F., J.Phys. Condens. Matter 141957 (2002). 